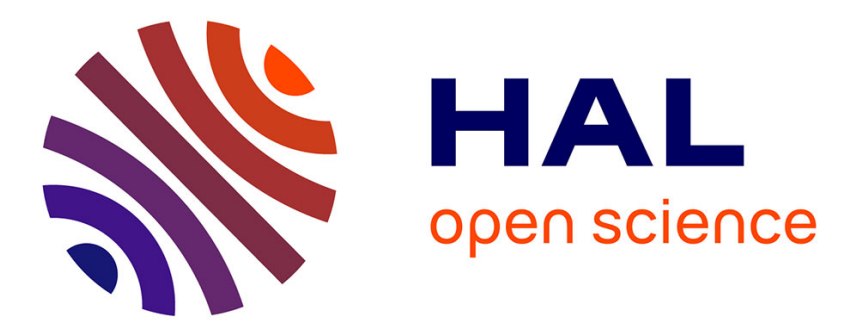

\title{
Formations sédimentaires et évolution de la vallée de la Deûle depuis le Pléniglaciaire supérieur à Houplin-Ancoisne (Nord de la France)
}

Laurent Deschodt, Pierre-Gil Salvador, Muriel Boulen

\section{- To cite this version:}

Laurent Deschodt, Pierre-Gil Salvador, Muriel Boulen. Formations sédimentaires et évolution de la vallée de la Deûle depuis le Pléniglaciaire supérieur à Houplin-Ancoisne (Nord de la France). Quaternaire, 2004, 15 (3), pp.269 - 284. 10.3406/quate.2004.1774 . hal-01489463

\section{HAL Id: hal-01489463 https://hal-inrap.archives-ouvertes.fr/hal-01489463}

Submitted on 3 Mar 2019

HAL is a multi-disciplinary open access archive for the deposit and dissemination of scientific research documents, whether they are published or not. The documents may come from teaching and research institutions in France or abroad, or from public or private research centers.
L'archive ouverte pluridisciplinaire HAL, est destinée au dépôt et à la diffusion de documents scientifiques de niveau recherche, publiés ou non, émanant des établissements d'enseignement et de recherche français ou étrangers, des laboratoires publics ou privés. 


\title{
FORMATIONS SÉDIMENTAIRES \\ ET ÉVOLUTION DE LA VALLÉE DE LA DEÛLE DEPUIS LE PLÉNIGLACIAIRE SUPÉRIEUR A HOUPLIN-ANCOISNE (Nord de la France)
}

\author{
Laurent DESCHODT*, Pierre-Gil SALVADOR** et Muriel BOULEN***
}

\begin{abstract}
RÉSUMÉ
Dans la région d'Houplin-Ancoisne (vallée de la Deûle, Nord), l'ampleur de la sédimentation loessique du Pléistocène supérieur dans le fond de vallée a favorisé la conservation des dépôts fluviatiles antérieurs, sur une bande de plus de $3 \mathrm{~km}$ de large et parfois jusqu'à $22 \mathrm{~m}$ d'épaisseur. Un transect partiel de la vallée de la Deûle a révélé un chenal tardiglaciaire qui incise sur environ $3 \mathrm{~m}$ les dépôts antérieurs, créant une terrasse Plésitocène supérieur / Tardiglaciaire. Son remblaiement débute précocément au Bølling, mais la majeure partie du comblement est datée du Dryas récent : après un dépôt lité relativement riche en débris végétaux (environ $1 \mathrm{~m}$ ), la partie supérieure $(0,9 \mathrm{~m})$ est azoïque et s'accumule rapidement (après 12350 -12150 cal. BP). Ce comblement terminal du Dryas récent témoigne d'une relative sécheresse et d'une modification de la morphologie du chenal. L'incision holocène, décalée par rapport au chenal tardiglaciaire, est plus modérée. Elle dégage une morphologie de basses terrasses creusées dans les nappes fluviatiles antérieures et le loess qui les scelle. Après un dépôt grossier, la tourbe se développe à partir du Boréal, déborde du lit mineur et ennoie les zones les plus basses du fond de vallée. La palynozone atlantique repérée dans la tourbe offre l'image d'un paysage boisé dominé par le Tilleul avec des traces d'anthropisation fugaces. Depuis le Néolithique, la micro-topographie du bas de versant à proximité de la rive droite est peu à peu régularisée par les colluvions.
\end{abstract}

Mots-clés : Deûle, Tardiglaciaire, Dryas récent, Atlantique, dynamique fluviale, Nord de la France.

\section{ABSTRACT}

SEDIMENTARY FORMATIONS AND EVOLUTION OF THE DEÛLE VALLEY SINCE THE UPPER PLENIGLACIAL AT HOUPLIN-ANCOISNE (Northern France)

In Houplin-Ancoisne area (Deûle valley, north of France), the size of the upper pleistocene loessic sedimentation in the bottom of the valley facilitated the former river deposits conservation, over more than a $3 \mathrm{~km}$ large belt, up to $22 \mathrm{~m}$ thick. A partial cross-section of the valley revealead a lateglacial channel which incises the former deposits on about $3 \mathrm{~m}$ depth creating an upper pleistocene terrace. Its filling begins earlier in the Bølling phase, but the main filling dates from the Younger Dryas phase : after a bedded deposit quite rich in vegetal remains (around $1 \mathrm{~m}$ ), the upper part $(0,9 \mathrm{~m}$ ) is azoïc and fast-setting (later 12350-12150 cal. BP). The final filling of the Younger Dryas gives evidence of a relative dryness and a modification of the channel morphology. The holocene incision, shifted from the tardiglacial chennal, is more moderate. It created low terraces digged into the frontal fluvial layers and the loess that fixes them in. After a coarse sedimentary deposit, the peat developps from the Boreal, overflows the mean bed and inundates the Valley lowest zone. Atlantic palynozone spoted in the peat gave a wooded landscape where lime trees prevail with fugace anthropogenic pollens. Since the Neolithic period, the microtopography of the bottom slope near the right bank is progressively sorted out by the colluviums.

Key-words : Deûle river, Lateglacial, Younger Dryas, Atlantic, fluvial dynamic, North of France.

\section{INTRODUCTION}

En contrebas de l'Artois, le « bas-pays » du nord de la France est parcouru de petits cours d'eau se dirigeant vers le nord (fig. 1). De superficie réduite, les bassins versants sont drainés par des rivières au débit limité, coulant sur des pentes faibles inférieures à $1 \%$ dès que l'on quitte les versants de l'Artois. Néanmoins, les vallées sont fréquemment très larges, jusqu'à former de vastes plaines humides, telles les plaines de la Lys $(20 \mathrm{~km}$ de large) et de la Scarpe (6 à $15 \mathrm{~km}$ de large). Cette inadéquation du calibrage des vallées s'explique par un dispositif hérité d'une tectonique de blocs (Colbeaux et al, 1978; Paepe, 1965 ; Sommé, 1977). La couverture loessique, qui

\footnotetext{
* INRAP Nord-Picardie, 518 rue Saint-Fuscien, 80000 AMIENS, E-mail : Ideschodt@nordnet.fr

** Université Lillel. UFR de Géographie. UMR 8141 " Géographie des Milieux Anthropisés ”, Av. Paul Langevin, 59655 VILLENEUVE D'ASCQ cedex. E-mail : pierre-gil.salvador@univ-lille.fr

*** INRAP Nord-Picardie, Centre de Recherches Archéologiques, Abbaye Saint-Jean-des-Vignes, 02200 SOISSONS, E-mail : inrap.soissons@wanadoo.fr
} 

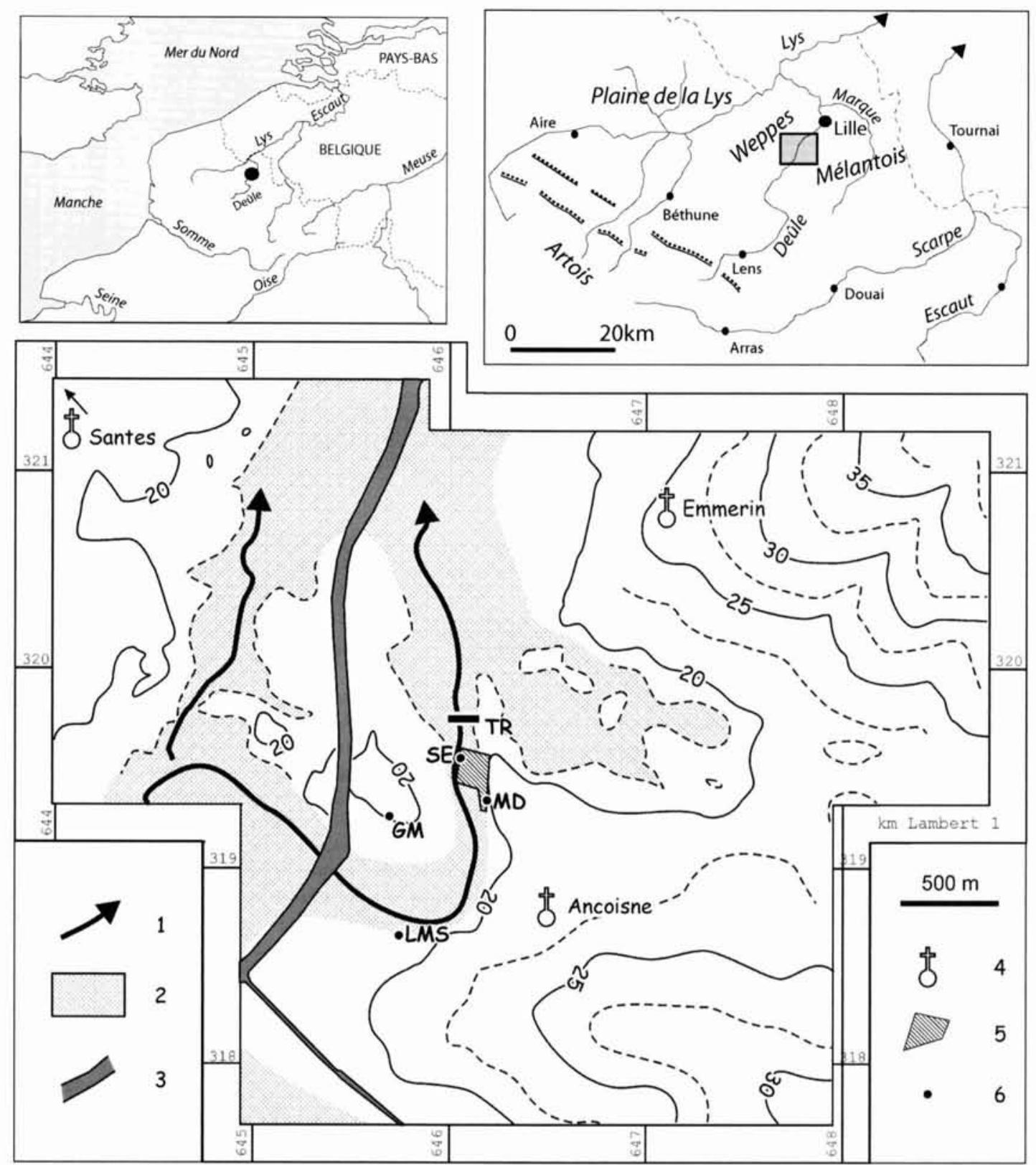

Fig. 1 : Localisation du secteur d'étude dans la vallée de la Deûle. 1 : ancien lit mineur holocène repéré sur carte topographique ; 2 : extension approximative des zones humides ; 3 : canal ; 4 : ville ; 5 : emprise de la station d'épuration ; 6 : localisation des sites d'observation, « TR " : transect tarière, «SE ", station d'épuration, « MD » rue Marx Dormoy, « GM » Guy Mocquet, « LMS ", le Marais de Santes.

Fig. 1: Location of the study area in the Deule valley. 1: ancient Holocene low channel located on topographic map; $2:$ approximate extension of the wetland; 3: channel; 4: city; 5; extension of the filtering station; 6: location of the sites.

les a envahies à la fin du Pléistocène supérieur, renforce l'empâtement de reliefs déjà modérés. Le bas-pays se caractérise également par l'artificialisation intense de son réseau hydrographique.

Affluent de la Lys, la Deûle est représentative des rivières drainant cette région. Dans la zone d'étude, elle emprunte un fond de vallée d'environ $2 \mathrm{~km}$ de large qui est disproportionné par rapport aux données hydrologiques : le bassin versant totalise seulement $900 \mathrm{~km}^{2}$ dont $500 \mathrm{~km}^{2}$ en amont de la zone d'étude et le débit interannuel de la rivière se limite à $5 \mathrm{~m}^{3} / \mathrm{s}$ juste à l'amont de Lille (Agence de l'eau, 2002). Bien que moins spectaculaire que dans les plaines voisines de la Lys et de la Scarpe, l'influence de la tectonique est sensible. Les variations de largeur du fond de vallée de la Deûle sont conformes à la structure dont l'influence se manifeste sous la forme d'un rétrécissement à $400 \mathrm{~m}$ au droit de l'axe synclinal du Mélantois (commune d'Haubourdin),
$3 \mathrm{~km}$ en aval de la zone d'étude. Conjointement, l'amincissement du manteau alluvionnaire et la disparition des tourbes permet d'envisager une tectonique récente (Sommé, 1967, 1977). A l'aval de cet axe, les épaisseurs d'alluvions augmentent considérablement pour atteindre 30 mètres à la confluence avec la Marque (Sommé, 1967). La tendance à la subsidence, la largeur de la vallée, la faiblesse des pentes et l'abondance de la couverture limoneuse susceptible d'être facilement incisée et mobilisée par la rivière confèrent à ce fond de vallée un fort potentiel d'enregistrement sédimentaire. S'ajoute à ces caractéristiques naturelles une anthropisation du réseau hydrographique particulièrement marquée : les nombreux aménagements hydrauliques et les canalisations successives réalisées depuis le Moyen Age tendent à effacer la perception du cours holocène primitif.

Les environnements tardiglaciaires et holocènes de la vallée de la Deûle ont été en premier lieu appréhendés 
à travers les travaux synthétiques de J. Sommé (1977). Aujourd'hui, les recherches accompagnent les fouilles archéologiques. Une première synthèse paléogéographique de la vallée à la hauteur de l'agglomération lilloise a été proposée par L. Deschodt (1999). Une étude a également été menée à grande échelle dans la partie amont, sur le site de Dourges, lors des investigations archéologiques réalisées dans l'emprise de la " plate-forme multimodale » (Teheux et Deschodt, 2000). D'autres travaux ont conduit à l'établissement de nouvelles stratigraphies à échelle locale (voir infra). L'objet de cet article est de synthétiser une série d'observations stratigraphiques réalisées entre 1999 et 2003 dans le secteur d'HouplinAncoisne afin de contribuer à une meilleure connaissance de la paléodynamique fluviale de la Deûle depuis le Tardiglaciaire.

\section{1 - DESCRIPTION DU SECTEUR D'ÉTUDE}

\section{LA VALLÉE DE LA DEÛle À HOUPLIN- ANCOISNE}

A Houplin-Ancoisne, la vallée de la Deûle s'étale sur une largeur de 2 à $3 \mathrm{~km}$, à une cote de $18-19 \mathrm{~m} \mathrm{~N}$.G.F (fig. 1). Elle sépare deux unités de reliefs modérés : à l'ouest le Pays de Weppes, caractérisé par un substrat tertiaire argileux et sableux (Landénien) avec une couverture loessique épaisse en bas de versant et, à l'est, le Mélantois, qui forme un dôme crayeux à couverture loessique d'ordre métrique (Sommé, 1977). La transition entre les versants et le fond de vallée est insensible, rendant incertain le tracé précis de leurs limites (Franchomme, 2003). Avant l'aménagement de la rivière, à partir du XIII siècle, le fond de vallée se présentait sous la forme d'un lacis de chenaux et de tourbières (Derville, 1988, 1990 ; Dessaux et al., 1998). La microtopographie est complexe : elle juxtapose les traces d'anciens lits fluviaux isolant des reliefs au sein de la vallée (fig. 1).

\section{LA STRATIGRAPHIE DE LA VALLÉE D'APRÈS LES DONNÉES GÉOTECHNIQUES}

La coupe stratigraphique générale de la vallée a été construite à partir de 27 sondages géotechniques répertoriés par les services du B.R.G.M. et du C.E.T.E (fig. 2). Elle recoupe le fond de vallée et les versants selon un axe ONO-ESE dominant. Les alluvions quaternaires, essentiellement sableuses, se développent sur une épaisseur croissant vers l'ouest, de $5 \mathrm{~m}$ à $10-15 \mathrm{~m}$ en moyenne. Elle atteint localement $22 \mathrm{~m}$, en raison d'un surcreusement abaissant le toit de la craie à $-3 \mathrm{~m}$ N.G.F. Le contact avec le substratum est aisé à suivre sur une bonne partie de la coupe, quand il s'opère avec la craie, mais il devient incertain à l'ouest au contact des formations tertiaires argilo-sableuses. Les versants de la vallée s'ouvrent dans des limons loessiques pléniglaciaires qui masquent sur les deux rives la limite des alluvions. Le chenal tardiglaciaire-holocène analysé dans cet article (chenal 1) est incisé dans les formations fluviatiles weichséliennes et les limons loessiques qui les recouvrent partiellement. Un second chenal, encore non exploré, se développe plus à l'ouest à l'aplomb du surcreusement maximal (chenal 2).

\section{LES OBSERVATIONS DE TERRAIN}

A l'occasion d'opérations archéologiques, une série d'observations à la pelle mécanique ont été effectuées dans et à proximité de l'ancien lit mineur historique de la Deûle (fig. 1). Il s'agit des sondages en puits de la station d'épuration ("SE »; Deschodt et Créteur, 1999), du décapage de la rue Marx Dormoy ( $(\mathrm{MD}$ »; Martial, étude en cours), d'un décapage sur le lieu-dit « Le Marais de Santes » («LMS "; Deschodt, 2003) et des sondages de la rue Guy Mocquet ( GM »; Martial, 2003). Ces observations sont complétées par un transect partiel réalisé à la tarière à main et positionné directement au nord de la station d'épuration d'Houplin-Ancoisne (« TR », fig. 1 et fig. 3). La synthèse des données est structurée à partir des résultats apportés par le transect.

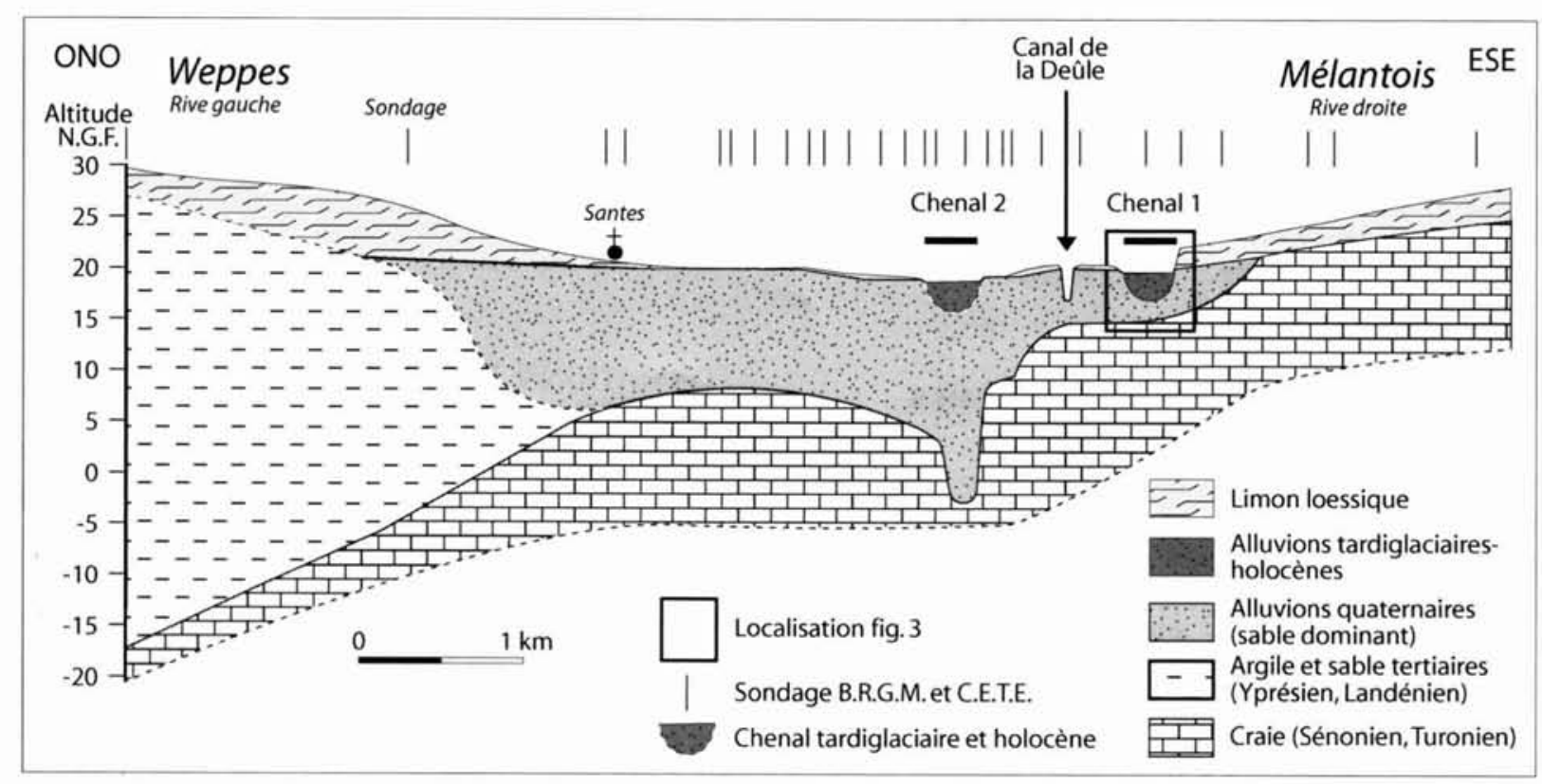

Fig. 2 : Transect à travers la vallée de la Deûle à Houplin-Ancoisne, d'après les sondages géotechniques. Fig. 2: Cross-section through the Devile valley at Houplin-Ancoisne, according to the geotechnics cores. 


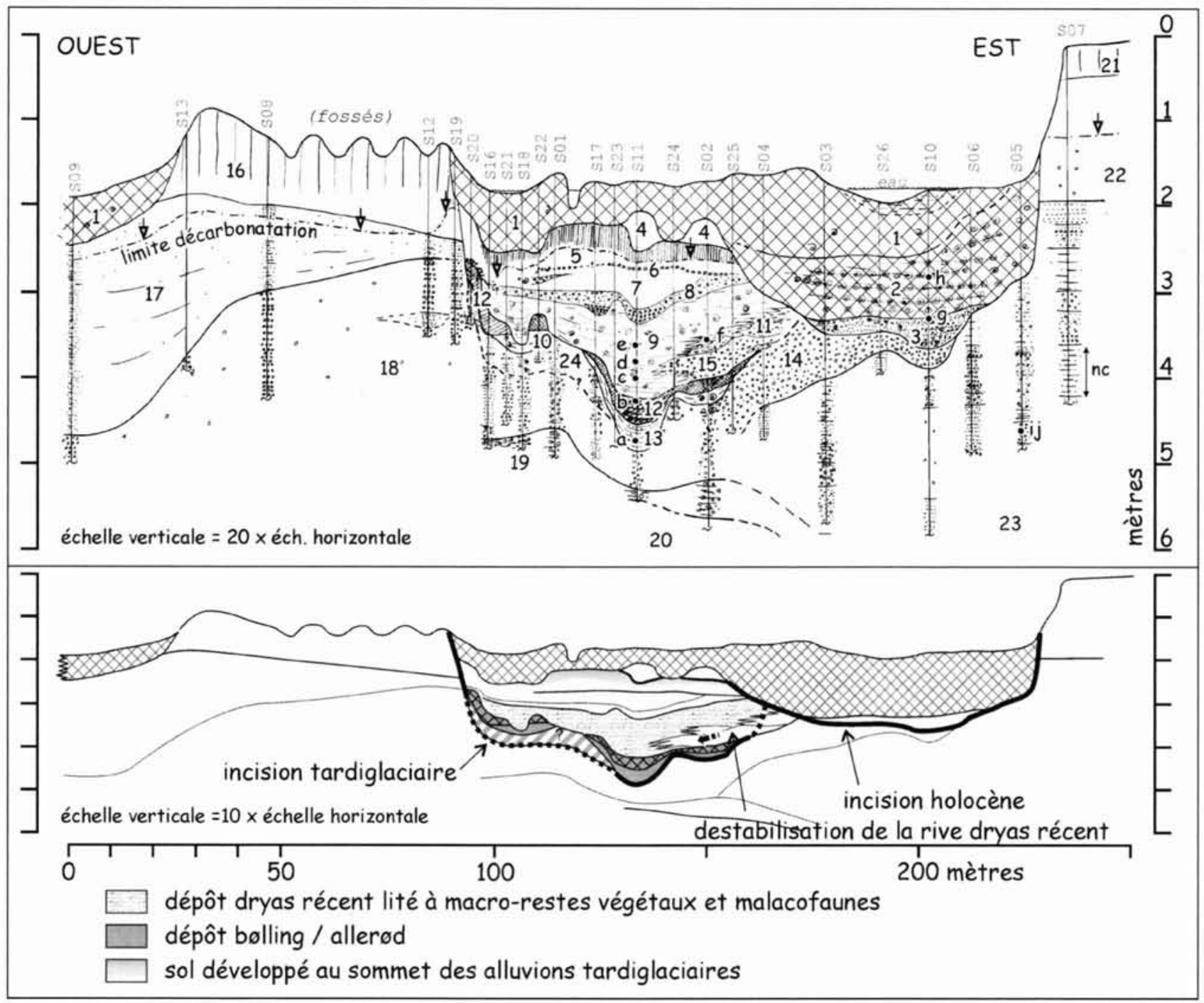

Fig. 3 : Transect partiel du fond de vallée. 1 : limon gris sombre très organique ; 2 : limon brun tourbeux avec faciès lité et malacofaunes abondantes ; 3 : sable lité gris avec craie et malacofaunes brisées ; $4:$ limon argileux ocre ; $5:$ limon bleu calcaire avec développement d'un sol ; 6 : limite érosive avec un lit de graviers de craie non usés; $7:$ limon gris calcaire ; $8:$ limon sableux à graviers; 9 : limon et limon sableux lité avec malacofaune et débris végétaux ; 10 : limon plastique verdâtre ; 11 : sable limoneux calcaire gris, lité, avec petits bois flottés ; 12 : limon organique lité, malacofaune, macrorestes végétaux, passant latéralement à une tourbe franche ; 13 : limon et limon sableux lité, malacofaune et restes végétaux ; 14 : gravier de craie roulé ; 15 : graviers de craie sans matrice; 16 : limon loessique non calcaire avec développement d'un sol brun ; 17 : lits fins et réguliers de limon et de sable ; 18 : gravier de craie roulé, litages ; 19: gravier et blocs de craie parfois anguleux à matrice limoneuse ; 20 : craie fragmentée en place ; 21 : labour; 22 : limon loessique pédogénisé au sommet avec gravier de craie et « poupées » à la base ; 23 : série très hétérogène, avec limon, sable, gravier (nc=limon sableux non calcaire) ; 24 : série hétérogène, sablo-graveleux avec passages de limon plastique.

Fig. 3: Partial cross-section of the valley. 1: dark gray organic silt; 2: laminated brown organic silt with abundant malacofaunas; 3: laminated grev sand with chalk and broken malacofaunas; 4: ochre clayey silt; 5: blue calcareous silt with soil; 6: erosion surface with a non eroded chalky gravel bed; 7: grey calcareous silt; 8: sandy silt with gravels; 9: laminated silt and sandy silt with malacofaunas and plant remains; 10: green silt; 11: grey calcareous silty sand, with little wood remains; 12: laminated organic silt, malacofaunas, plant macroremains, being transformed laterally into peat: 13: silt and laminated sandy silt, malacofaunas and plant remains; 14: rolled chalky gravels; 15: chalky gravels; I6: non calcareous loessic silt with brown soil; 17: fine regular beds of silt and sand; 18: rolled chalky gravel, laminations; 19: gravels and angular chalk boulders with silty matrix; 20: fragmented chalky substratum; 21: ploughing; 22: pedogeneous loessic silt at the top with chalky gravels at the base; 23: heterogeneous layers with silt, sand and gravel ( $n c=$ non calcareous sandy silt); 24: heterogeneous layers with sandy gravels and silt.

\section{2 - LES FORMATIONS ANTÉRIEURES AU TARDIGLACIAIRE}

\section{SUBSTRAT CRAYEUX}

Une craie massive (unité 20, fig. 3), probablement la craie fragmentée en place, a été repérée à la base des sondages S2 et S11 (fig. 4). Elle est surmontée par une couche de fragments de craie anguleux de taille centimétrique (unité 19), pris dans une matrice de limon crayeux. La topographie du contact entre la craie et les formations alluviales présente une légère pente vers l'est.

\section{FORMATIONS FLUVIATILES ANTÉRIEURES AU DÉPÔT DE LOESS}

Tous les sondages ont rencontré des sédiments lités (unités 14, 15, 17, 18 et 23) antérieurs au dépôt loessique (16 et 22). A l'Ouest, il s'agit de lits de graviers de craie roulés (18), sans matrice ou avec un peu de sable, recouverts de fins lits de sable et de limon (17). Vers l'est, les faciès sont beaucoup plus diversifiés - lits de sable, de graviers de craie, passages limoneux (23) - à tel point qu'il n'est pas possible d'établir de corrélations entre sondages. Une datation effectuée dans l'unité 23 («ij ", fig. 3) a donné une date aberrante 


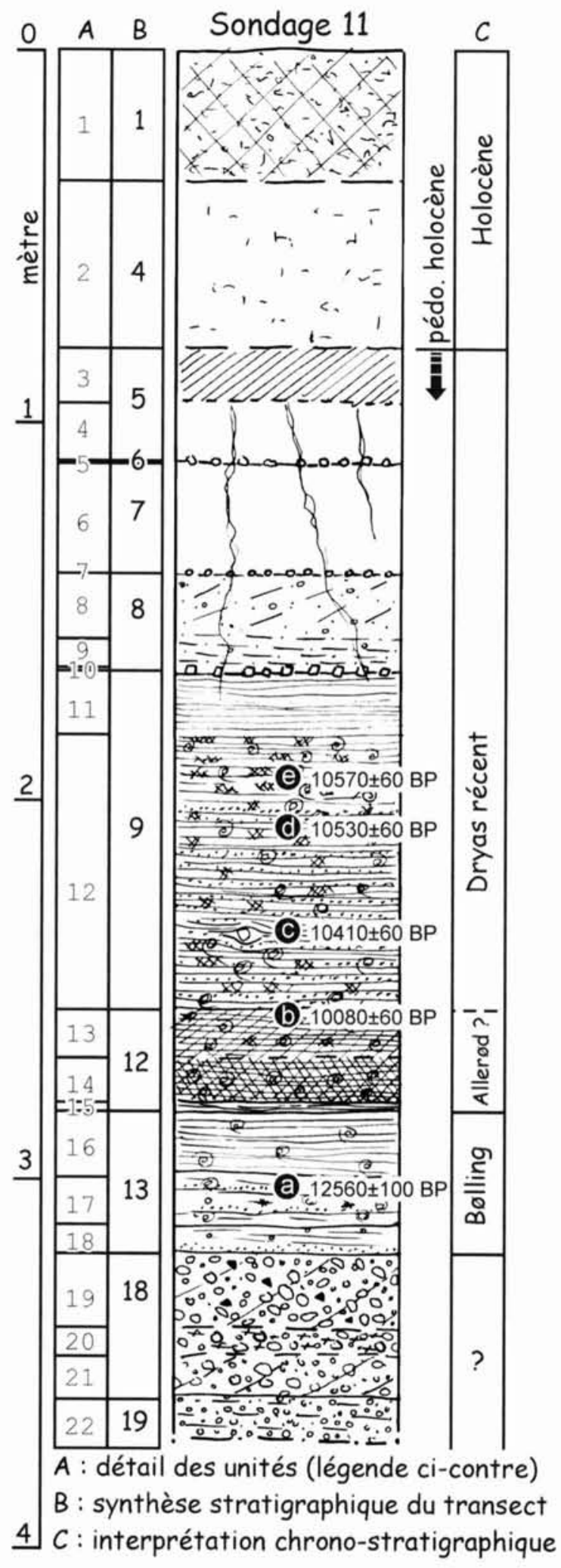

(4135 $\pm 40 \mathrm{BP})$ pouvant provenir d'une racine ou d'une pollution du sondage.

Le transect illustre la complexité et l'extension des alluvions antérieures au dépôt loessique. Les sédiments observés constituent une faible part d'un remblaiement complexe se développant sur plus de $2 \mathrm{~km}$ de large et jusqu'à $20 \mathrm{~m}$ de profondeur (fig. 3). A Lille, deux talwegs sont inscrits dans la craie sous la cote 0 N.G.F. Le tracé occidental est scellé par les formations de versant saaliennes (Sommé, 1969, 1977). Le remblaiement de la vallée est donc lié à une histoire longue débutant avant le Saalien (Sommé, 1977). Les faciès sont essentiellement sableux. Les mentions de dépôts organiques voire tourbeux sont fréquents, à des profondeurs variables (Ladrière, 1886 ; Sommé, 1977 ; Deschodt, 1999). A défaut d'étude locale précise sur ce remblaiement ancien, une comparaison peut être établie avec le sondage d'Erquinghem, dans la région proche de la plaine de la Lys (Sommé et al., 1996). Le remblaiement y débute à 5 mètres sous la cote 0 par une séquence tourbeuse éemienne, suivie par une séquence fluviatile limonosableux attribuée au Pléniglaciaire weichsélien. Une rémission de l'activité fluviatile, une influence éolienne notable et une légère pédogenèse (niveau organique, débris de végétaux) attribuée à un interstade du Pléniglaciaire sont perçues sous la séquence fluviatile tardiglaciaire-holocène.

FORMATIONS LOESSIQUES DE FOND DE VALLÉE

Les formations alluviales sont recouvertes par un limon loessique (unités 16 et 22 , fig. 3 ) en continuité avec la couverture limoneuse du versant. Le recouvrement pléistocène fluviatile par des sédiments éoliens loessiques est

— Fig. 4: Stratigraphie détaillée du sondage 11. 1 : limon brun sombre tourbeux, structure " granuleuse ", compressible ; 2 : limon argileux ocre à marbrures rouilles, structuré ; 3 : limon gris ; 4: limon bleu à traces rouilles ; 5 : lit de gravier de craie légèrement usé ; 6 : limon gris clair ; 7 : gravier roulé $; 8$ : limon sableux à points de craie $(<1 \mathrm{~mm}) ; 9$ : id., mais plus sombre et lits fins nettement visibles ; 10 : limite nette marquée par des graviers de craie anguleux ; 11 : limon clair, très peu cohérent avec fines lamines ; 12 id., en plus sombre avec malacofaune et débris végétaux abondants, texture sableuse vers $204 \mathrm{~cm}$, un cailloux de craie légèrement usé à $235 \mathrm{~cm}$ avec charbons et bois flottés; 13 : limon organique brun à noir lité, lits de malacofaune, charbons, débris végétaux ; 14 : limon tourbeux brun gris, micro-laminé, malacofaune et débris de végétaux ; 15 : alternance de limon tourbeux et de limon gris-verdâtre : 16 : lamines de limon gris verdâtre et malacofaune, moins abondante ; 17 : limon clair, plastique avec quelques lamines sableuses, de 307 à $313 \mathrm{~cm}$, lamines soulignées par micro-charbons de bois ; quelque malacofaune et gravier de craie ; 18 : limon très plastique, très clair et légèrement verdâtre, graviers de craie épars, lamines sableuses vers $318 \mathrm{~cm} ; 19$ : gravier de craie usé, très hétérométrique dans une pâte crayeuse, quelques silex sub-sphériques (environ $1 \mathrm{~cm}$ ) usés et éolisés ; 20 : id., mais avec vides et revêtement d'oxyde ferrique ; 21 : présence d'un galet $(5 \mathrm{~cm})$ de craie légèrement usé dans cailloutis hétérométriques ; 22 : limon un peu sableux à graviers de craie abondant.

Fig. 4: Detailed stratigraphy of core 11. 1: dark brown organic silt; 2: ochre clayey silt with iron staining; 3 : grey silt; 4 : blue silt with iron staining; 5 : chalk gravel bed slightly eroded: 6 : light grey silt; $7:$ rolled gravel; 8: sandy silt with scattered chalk grains; $9:$ id., but darker and more visible fine beds; 10: clear limit marked by angular chalk gravels; 11: light silt with fine laminations: 12: id. but darker with malacofaunas and abundant plant remains, sandy texture around $204 \mathrm{~cm}$. granule of chalk to $235 \mathrm{~cm}$ with charcoals and floated wood: 13: brown to black laminated organic silt, malacofaunas beds, charcoals, plant remains; 14: micro-laminated brown-grey organic silt, malacofaunas and plant remains; 15: alternation of organic silt and gray-green silt: 16: laminated grey-green silt and less abundant malacofaunas; 17: light clayey silt with some sandy laminations, from 307 to $313 \mathrm{~cm}$ laminations underlined by micro-charcoals of wood, some malacofaunas and chalk gravel; 18: clavey silt, very light and slightly greenish, scattered chalk gravels, sandy laminations around $318 \mathrm{~cm} ; 19:$ chalk gravel, very heterometric in a chalky matrix, some sub-spherical flints (approximately I cm) eroded and eolized; 20: id., but with vacuums and oxide ferric: 21 : presence of a chalk pebble (5 cm) slightly eroded in gravel heterometric matrix: 22 : sandy silt with abundant chalk gravels. 
fréquent dans les fonds de vallée du nord-est de la France (Antoine, 1997b ; Antoine et al., 2003). Dans la vallée de la Deûle, ces formations de recouvrement ont une très grande extension. Elles se distinguent par une altitude relative élevée, en position de basse terrasse par rapport aux formations holocènes (Deschodt, 1999).

La couverture limoneuse supporte la pédogenèse tardiglaciaire-holocène qui se traduit par une décarbonatation, une coloration brun-orangé du sédiment accompagnée d'accumulations d'oxydes de manganèse (horizons B). Les observations directes de la partie non atteinte par les interventions anthropiques (fossés en rive gauche du transect) ou par la pédogenèse montrent un faciès lité comportant parfois des lits de sable ou de graviers de craie (fig. 5) (Deschodt, 1999, 2003 ; Deschodt in Martial, 2003). La partie supérieure est essentiellement limoneuse avec en alternance des lits centimétriques de limon calcaire blanchâtre. Les séries de lits sont parallèles mais fréquemment plissées, voire ondulées, avec de fréquentes petites fentes de dessiccation syngénétiques. A Lille, des faciès similaires mais avec des lits extrêmement calcaires, ont pu être suivis sur plusieurs centaines de mètres (Lille "Anciens abattoirs/Quais du Vieux Lille » ; Deschodt, 1999). Plusieurs auteurs ont remarqué et décrit avec précision ce faciès particulier qualifié d'" argile blanchâtre " ou de " limon crayeux » et que l'on observe sur une vaste aire au nord de la ville (depuis la Citadelle jusqu'à la Place du Concert). Les lits, « ondulés » ou "plissés ", composent des « faisceaux de veines parallèles », gardent un " parallélisme relatif », sont " stratifiés avec une régularité parfaite» (Ladrière, 1885 ; Hette, 1886 ; Théry in Révillion, 1988 ; Vallin, 1991). Mais ils peuvent aussi s'entrecroiser selon un angle faible et des discordances interviennent entre faisceaux de lits (photo 1). Aucune coupe n'a encore mis en évidence une transition latérale avec un chenal contemporain bien marqué à remplissage différencié. A Houplin comme à Lille, la séquence limoneuse litée est épaisse de 1 à 2 mètres. Elle s'enrichit de quelques graviers de craie et de lits sableux, épisodiques dans la partie supérieure du dépôt mais plus fréquents vers le bas, où ils peuvent êtres très régulièrement répétés. Quand le sédiment est exclusivement limoneux et très calcaire, sa décarbonatation ultérieure rend alors illisible la structure. La séquence

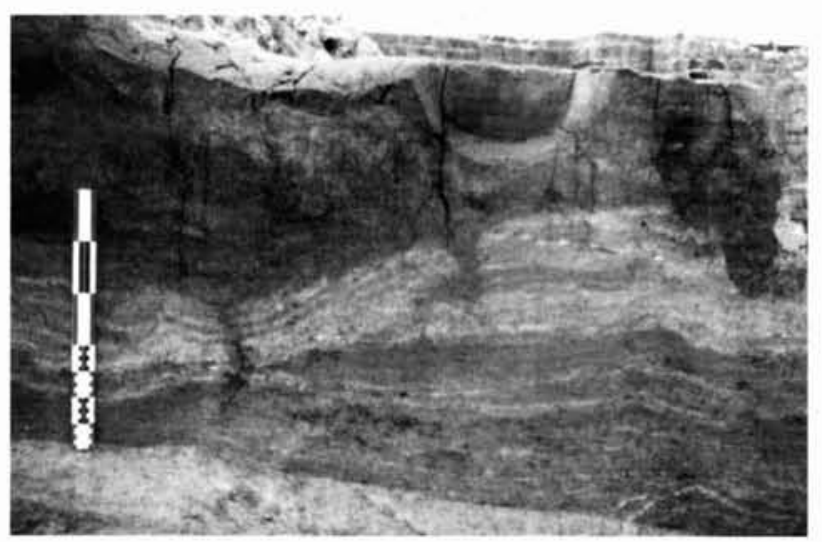

Photo 1 : Loess lité (chantier « Quais du Vieux Lille », 1997, photo D. Bossut, INRAP, échelle $0,5 \mathrm{~m}$ ).

Photo 1: Bedded loess, scale $0.5 \mathrm{~m}$. ainsi décrite repose sur les formations fluviatiles sableuses ou sablo-graveleuses, parfois avec un contact érosif lié au développement de petites ravines en pied de versant (fig. 5).

La description des sables de couvertures (alternances limon/sable ou sable fin/grossier), en particulier le faciès " alternating bedding " ou " wet aeolian ", avec leurs structures cryogéniques associées (Schwan, 1986; Kasse, 1997), peut tout à fait s'appliquer à la formation loessique du fond de vallée de la Deûle, si ce n'est, dans ce cas, le caractère très calcaire du sédiment et sa texture beaucoup plus fine. Ces deux traits peuvent toutefois s'expliquer par l'influence du substrat local et par le granoclassement éolien : la zone de transition entre les sables de couverture et le loess s'interrompt à quelques kilomètres vers le nord et l'ouest contre l'obstacle formé par le Pays de Weppes, à seulement 5 kilomètres en aval de Lille (Paepe et Vanhoorne, 1967 ; Paepe et Sommé, 1970). De même, dans un contexte plus sableux, de nombreuses vallées de Belgique, des Pays-Bas et d'Allemagne offrent des faciès similaires à la fin du Pléniglaciaire supérieur (Vandenberghe, 1985 ; Vandenberghe, 1995 ; Huisink, 2000 ; Van Huissteden et Vandenberghe, 1988 ; Mol, 1997 ; Kasse et al., 2003). Les alluvions y ont enregistré une prépondérance croissante des processus éoliens au cours de la fin du Pléniglaciaire supérieur.

A Quesnoy-sur-Deûle, à quelques kilomètres en aval de la zone d'étude, à l'entrée dans la Plaine de la Lys et en rive gauche du cours holocène, les formations fluviatiles sableuses sont affectées par un pseudomorphe de grande fente de gel puis recouvertes par environ 2 mètres de lits sablo-limoneux (Sommé, 1977). Ceux-ci sont interprétés selon la synthèse chrono-stratigraphique de Paepe et Sommé (1970) comme des « sables et limons de couverture " et correspondent au faciès typique de la zone de transition. Ils sont attribués au Pléniglaciaire supérieur, postérieurement à l'horizon à coins de glace le plus récent « Nagelbeek/Kesselt » (Lautridou et Sommé, 1981 ; Antoine et al., 1998). Nous proposons une corrélation entre la séquence loessique lité du fond de vallée de la Deûle (en amont de la plaine de la Lys) et la couverture sableuse litée plus septentrionale déposée sous commande éolienne. Dans la synthèse de Paepe et Sommé (1970), elle correspondrait à l'unité litho-stratigraphique du « Limon de couverture 2 ».

Pendant la première partie du Pléniglaciaire supérieur, la forte dynamique saisonnière des rivières a remanié le loess contemporain déposé dans la bande active. Il se peut que le limon mis en suspension ait pu être parfois intégré aux alluvions grossières et composer ces faciès à lits de limons fins et sables que l'on observe dans la vallée de la Deûle (faciès SLL, fig. 5). Par la suite, la mise en place des formations presque exclusivement limoneuses (faciès LL, fig. 5) dans tout le fond de vallée implique une réduction considérable de la dynamique fluviale. Elle peut résulter d'une aridification du climat et de la dégradation du pergélisol, qui permet de nouveau l'infiltration des eaux de fontes. Ces modalités contribuent ainsi à une réduction importante des écoulements allant jusqu'à leur disparition dans les vallées et vallons de rang inférieur (Kasse, 1997 ; Vandenberghe, 2001 ; Vandenberghe et 


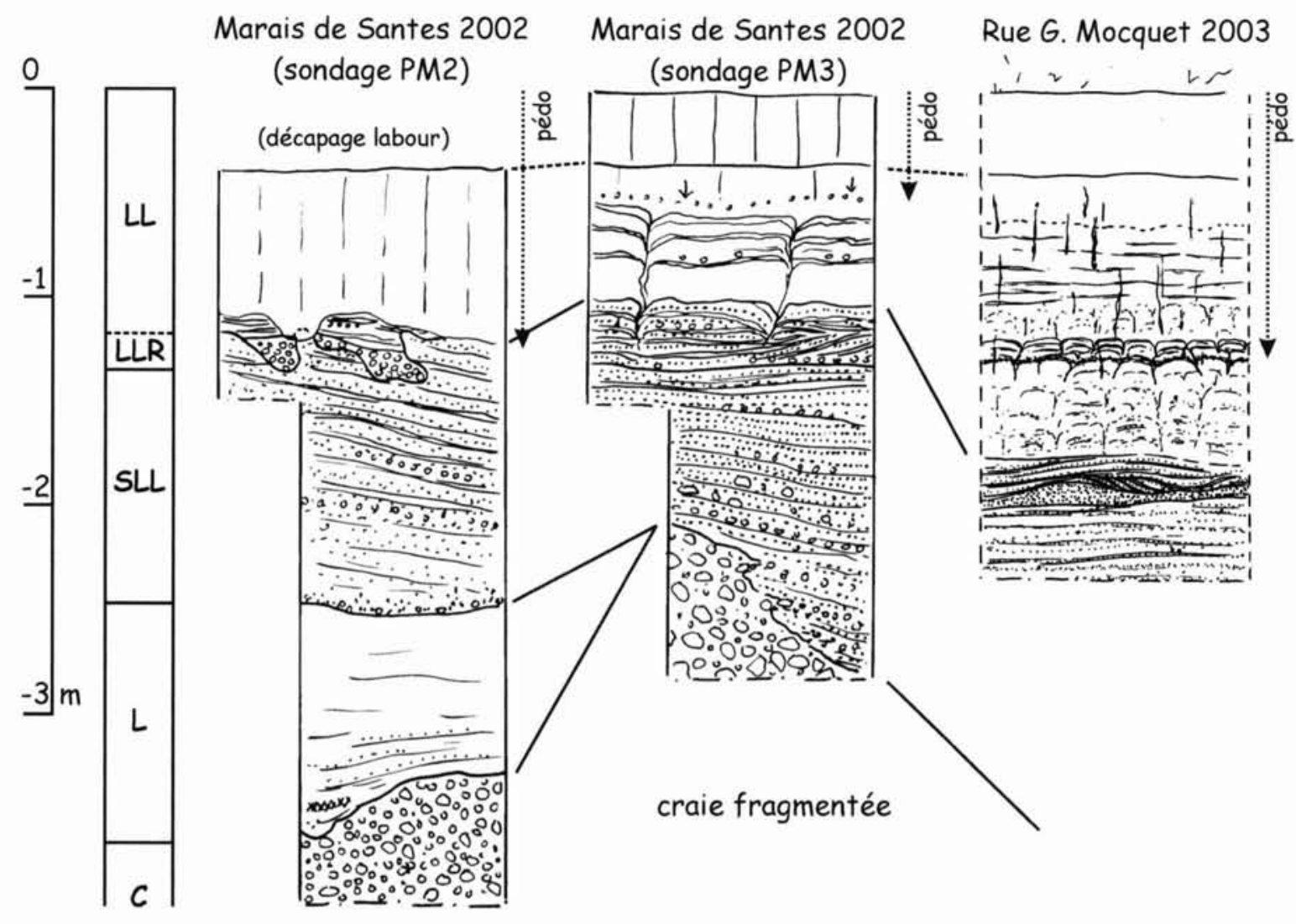

Fig. 5 : Observations en puits de formations loessiques de fond de vallée. LL : limon loessique lité, affecté par la pédogenèse tardiglaciaireholocène (lits de limon très calcaires blancs sous la limite de décarbonatation, quelques lits plus sableux, fentes de dessications syngénétiques) ; LLR : limon loessique lité comblant de petites ravines creusées dans le sédiment sableux sous-jacent (uniquement perçu dans le sondage PM2); SLL : sable et limon lité, graviers de craie abondants; $L$ : limon fin de décantation, finement lité (comblement de chenal pléistocène) ; $C$ : craie fragmentée hétérométrique.

Fig. 5: Observations of loess formations in the vallev, according to a trench. LL: laminated loessic silt, affected by the Lateglacial-Holocene pedogenesis (beds of white calcareous silt under the decarbonation limit, some sandier beds, syngenetic shrinkage crack): LLR: laminated loessic silt filling of small gullies dug in the subjacent sandy sediment (only observed in core PM2); SLL: sand and laminated silt, abundant chalk gravels; L: fine silt of decantation, finely laminated (filling of pleistocene channel); C: fragmented heterometric chalk.

Woo, 2002). Aux Pays-Bas, elles sont considérées comme essentielles au dépôt des sables de couvertures (Kasse, 1997). Près du site d'étude, en rive droite de la Deûle, le vallon occupé aujourd'hui par le canal de Seclin semble conforme à ce schéma. Dans ce sous-bassin de $40 \mathrm{~km}^{2}$ ouvert majoritairement dans le substrat crayeux, des dépôts grossiers à graviers de craie sans matrice associés à un style fluvial tressé surmontent des formations limoneuses à passages organiques. Elles indiquent l'occurrence d'écoulements de forte compétence (Deschodt in Blondiau, 2002). L'ensemble est recouvert d'une couverture loessique homogène qui témoigne à l'inverse de la considérable réduction, sinon de l'arrêt, des écoulements provenant du Mélantois. L'enfouissement d'un volume important d'alluvions grossières avec leurs vides inter-granulaires a engendré un écoulement par la nappe très efficace, au détriment des écoulements de surface.

A la fin du Pléniglaciaire supérieur, les modalités suivantes étaient réunies dans la vallée de la Deûle : (a) un fond de vallée large et une pente longitudinale faible ; (b) un débit très limité en volume et probablement circonscrit au dégel estival ; (c) la poursuite d'une sédimentation loessique (Antoine, 2002), renforcée par les conditions topographiques locales de versant sous le vent; on observe ainsi jusqu'à plus de 4 mètres de loess postérieur à l'horizon repère en bas du versant du Weppes
(Sommé, 1968 ; Deschodt, 1996). De nombreux paramètres difficiles à appréhender interagissent de manière non linéaire sur le style fluvial tels le développement de la couverture végétale, la rapidité du dégel, la persistance éventuelle de la couverture neigeuse, de possibles barrage de neige, etc. (Vandenberghe, 2001; Vandenberghe et Woo, 2002). Les quelques chenaux ou amorces de chenaux observés à Lille sont très peu profonds et rapidement comblés (défluviations répétées ?). Sur le transect (fig. 3), une discontinuité de la couverture loessique entre les sondages 13 et 9 pourrait être héritée d'un de ces chenaux contemporains de la mise en place de la couverture loessique de fond de vallée. Le limon remanié par les eaux se dépose en lits sub-horizontaux. En fin de période hydrologiquement active, la persistance de flaques dans la plaine d'inondation ou de mouilles dans les petits chenaux a permis la concentration en carbonates des sédiments, sous la forme de lits plastiques et très calcaires (photo 1). L'émersion prolongée et le climat périglaciaire ont engendré le développement de nombreuses mais discrètes figures cryogéniques rapidement enfouies par l'inondation suivante. Les lits sableux, abondants vers la base de la séquence, disparaissent au fur et à mesure de l'exhaussement du fond de vallée. Ce classement granulométrique vertical témoigne de la faiblesse des apports de versants et de l'absence des remises en charge. Les 
alluvions anciennes sont pourtant proches et à la portée d'une incision un peu plus forte. La séquence a pris une extension importante au droit du site de la future ville de Lille où l'élargissement de la vallée a permis l'étalement de la crue printanière. Le caractère cyclique du dćpôt évoque une caractéristique de la sédimentation rapide lors des larges inondations des anabranching rivers périglaciaires (Vandenberghe, 2001).

Ce mode de remblaiement du fond de vallée dominé par une charge abondante d'origine ćolienne est commun à de nombreuses vallées situées au nord de la zone d'étude (cf. supra). A la même période, à la frontière belgo-nćerlandaise, la Mark se transforme en une large rivière en tresses incapable d'évacuer la charge éolienne sableuse (Vandenberghe et Bohncke, 1985 ; Vandenberghe et al.. 1987). Dans la vallée de la Haine, P. llacsaerts (1984) observe une migration des chenaux à colmatage sableux puis leur recouvrement progressif par des colluvions sablo-limoneuses provenant des versants. Par contre, les comparaisons sont plus difficiles à trouver avec le Bassin parisien où l'évolution des cours d'cau à la fin du Pléniglaciaire et à la transition avec le Tardiglaciaire est encore mal cernée (Pastre et al., 2000, 2002). Le recouvrement des nappes graveleuses par des dépôts loessiques n'est pas systématique mais souvent mentionné, en particulier dans la vallée de la Somme: loess ou loess sableux du Pléniglaciaire supéricur sur les alluvions grossières weichséliennes (Antoine, 1997b ; Antoine et al., 2003). Les observations de faciès loessiques similaires à ceux rencontrés à IJouplin-Ancoisne sont rares. Dans la moyenne vallée de l'Oise (Pastre ef al., 2003), une formation régulièrement litée de sable et de sćdiments fins marneux présente la même structure caractéristique. Les auteurs expliquent sa mise en place par des inondations saisonnières à la transition Pléniglaciaire/ Tardiglaciaire et suggèrent qu'elle fut préservée des érosions ultérieures grâce à sa position dans un ćlargissement du fond de valléc. Le manque d'éléments de comparaison peut être expliqué par la persistance d'écoulements plus dynamiques ou par une charge d'origine colienne moindre, mais cette lacune est plus certainement liće à des difficultés d'observations (Pastre é al., 2000). L'absence d'individualisation des dépôts du Pléistocène supérieur sous la forme d'une basse terrasse implique deux conséquences : (a) la totalité des dépôts loessiques de fond de vallée est soumise aux incisions et aux migrations des cours tardiglaciaires-holocènes ; (b) quand ils ont été préservés de l'érosion, les dépôts pléniglaciaires sont scellés par les alluvions ultérieures, limitant les possibilités d'observation directe (absence d'affleurement ou proximité de la nappe phréatique).

\section{3 - LES FORMATIONS FLUVIATILES TARDIGLACIAIRES}

\section{1 - LITHOSTRATIGRAPHIE}

L'ensemble des formations fluviatiles attribuables au Tardiglaciaire est contenu dans un chenal partiellement recoupé sur sa rive droite par un second chenal d'âge holocène (fig. 3). La base du remplissage (unité 24) forme une couche hétérogène sablo-graveleuse. Elle inclut des passées de limon plastique renfermant parfois des restes végétaux et des malacofaunes. Ce niveau se distingue ainsi de l'unité 18 sous-jacente à graviers de craie homogène. L'unité 13 colmate la partie la plus profonde du chenal, et peut-être son prolongement en rive gauche (unité 10) sous la forme d'un limon plastique clair lité d'environ $40 \mathrm{~cm}$ d'épaisseur. Ce niveau est recouvert sur $45 \mathrm{~cm}$ d'épaisseur par un limon brun organique lité (unité 12), avec malacofaunes abondantes, bois et charbon de bois, qui passe latéralement à une tourbe franche (rive gauche). On observe ensuite $90 \mathrm{~cm}$ de sédiment lité (unité 9), limoneux et sableux, avec petits graviers, lits de sable et malacofaunes. Cette unité s'interstratifie en rive droite avec deux niveaux plus grossiers issus des formations pléistocènes. Il s'agit de l'unité 15, composée de graviers de craie et de l'unité 11 , constituée d'un sable limoneux lité. Le niveau 8 regroupe des dépôts relativement grossiers et hétérogènes, formés de limon sableux à graviers plus ou moins abondants, qui ravinent légèrement l'unité 9 dans l'axe des écoulements. Le comblement terminal du chenal est réalisé sur $85 \mathrm{~cm}$ d'épaisscur maximum par deux limons gris homogènes calcaires (unités 7 et 5) séparés par un niveau érosif à graviers de craic anguleux qui tronque les niveaux inféricurs. Un petit sol s'est développé à la surface de l'unité 5 qui clôt la séquence tardiglaciaire.

\section{2 - INTERPRÉTATION CHRONOSTRATIGRAPHIQUE}

Le cadre chronologicue est précisé par 6 datations radiocarbones obtenues sur des macrorestes de bois extraits de la tarière à gouge (tab. 1). Deux d'entres elles posent un probleme en terme de chronostratigraphic. La date « $\mathrm{b}$ » est plus récente que le groupe cohérent « $\mathrm{c}$, $\mathrm{d}$, e », même si un très léger chevauchement existe entre «b» et « $\mathrm{c} »$ en années cal. BP (2 sigma). II pourrait s'agir d'un bois entrainé vers le bas lors du forage, ce qui nous amène à ne pas prendre en compte cette date pour l'interprétation. Par ailleurs, la date « $f$ » en position latérale, apparaît plus récente que l'ensemble «c, d, e ", alors qu'elle se situe à une altitude voisine du bois "e ». Néanmoins, un faible recoupement chronologique existe en années cal. BP, l'intervalle à 2 sigma se réduisant à 12340-12177 cal. BP entre «e » et «f ». La date n'est donc pas aberrante et est intégrée à l'interprétation.

\section{L'incision tardiglaciaire et la formation d'une ter- rasse Pléistocène supérieur/Tardiglaciaire}

Le chenal entaille les dépôts pléistocènes. L'incision maximum se situe $4,6 \mathrm{~m}$ sous le sommet des dépôts loessiques de rive droite et $3,6 \mathrm{~m}$ sous ceux de rive gauche. Toutefois nous ne savons pas s'il s'agit là du niveau de départ puisque la topographie antérieure n'a pas été conservée. II est probable que le tracé tardiglaciaire du chenal ait été préparé par des écoulements contemporains des formations loessiques dans des chenaux peu profonds. La topographie de la base de l'unité 1, en rive gauche, entre les sondages 13 et 9 , est peut-être le témoin d'un modeste chenal antérieur au Bølling. Elle se situe 2,3 $\mathrm{m}$ au-dessus de l'incision maximum tardigla- 


\begin{tabular}{|c|c|c|c|c|c|c|c|}
\hline \multicolumn{3}{|c|}{ échantillon } & \multirow{2}{*}{\begin{tabular}{|l} 
num. labo \\
GrA-23294
\end{tabular}} & \multirow{2}{*}{$\frac{\text { années BP }}{12560+/-100}$} & \multirow{2}{*}{$\begin{array}{c}\begin{array}{c}\text { interval } 1 \text { sigma } \\
\text { (années cal. BP) }\end{array} \\
15468-14325\end{array}$} & \multirow{2}{*}{$\begin{array}{l}\text { interval } 2 \text { sigma } \\
\text { (années cal. BP) } \\
15604-14165\end{array}$} & \multirow{2}{*}{\begin{tabular}{|l}
$\begin{array}{l}\text { attributions } \\
\text { chronolgiques }\end{array}$ \\
$\begin{array}{l}\text { Bølling (pas de } \\
\text { pretraitement) }\end{array}$
\end{tabular}} \\
\hline fig5-a & $\begin{array}{l}\text { charbon } \\
\text { de bois }\end{array}$ & $\mathrm{Pr} 12, \mathrm{~S} 11-304 \mathrm{~cm}$ & & & & & \\
\hline fig $5-b$ & bois & Pr11, $511-257 \mathrm{~cm}$ & GrA-23291 & $10080+/-60$ & $11918-11344$ & $12115-11261$ & Date réjetèe \\
\hline fig5-c & bois & $\mathrm{P}+10.511-235 \mathrm{~cm}$ & GrA-23290 & $10410+/-60$ & $12636-12107$ & $12838-11935$ & Dryas récent \\
\hline fig5-d & bois & Pr9. S11-200 à $216 \mathrm{~cm}$ & GrA-23289 & $10530+1-60$ & $12826-12346$ & $12920-12116$ & Dryas récent \\
\hline fig5-e & bois & Pr8, S11-196 cm & GrA-23287 & $10570+/-60$ & $12848-12365$ & $12932-12177$ & Dryas récent \\
\hline fig5-f & bois & $\mathrm{Pr} 1, \mathrm{~S} 2-181$ à $190 \mathrm{~cm}$ & GrA-23284 & $10190+/-60$ & $12255-11694$ & $12340-11443$ & Dryas récent \\
\hline fig5-g & bois & Pr7. S10-146 à $149 \mathrm{~cm}$ & GrA-23190 & $8380+/-50$ & $9473-9303$ & $9522-9158$ & Boréal \\
\hline fig5-h & bois & Pr5, $\$ 10-107 \mathrm{~cm}$ & GrA-23286 & $3590+/-40$ & $3961 \cdot 3832$ & $4056-3729$ & Subboréal \\
\hline fig5-ij & bois & Pr4, $55-285 \mathrm{~cm}$ & GrA-23285 & $4135+1-\quad 40$ & & & date rejetée \\
\hline fig 6 & os & $\begin{array}{l}\text { observations } 1999, \\
\text { sondage } 1\end{array}$ & $\begin{array}{l}\text { GRA-19038 } \\
\text { (Lyon-1547) }\end{array}$ & $5480+/-70$ & & & \\
\hline
\end{tabular}

Tab. 1 : Analyses radiocarbone (calibration avec C ALIB rev: 4.3.).

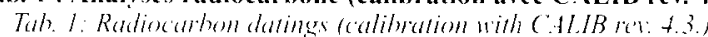

ciaire. Nous considérons qu'il s'agit de la valeur minimale de l'incision qui a dégagé une basse terrasse à travers l'abondant remblaiement éolien ou fluvio-éolien du fond de vallée, créant une morphologie similaire à celle de nombreuses rivières de Belgique et des Pays-Bas (Vandenberghe et Bohncke, 1985; Tebbens et al., 1999).

Une date réaliséc sur charbon à la base des dépôts $(12560 \pm 100 \mathrm{BP}, \mathrm{GrA}-23294$, tab. 1$)$ indique un colmatage précoce du chenal au Bølling, ce qui place probablement l'incision au tout début du Tardiglaciaire, bien que l'on n'ait pas localement de terminus post-quem précis pour le dépôt des loess du Pléniglaciaire supérieur. $\mathrm{Ce}$ dernier est identifié vers 15-16 k BP dans les régions voisines de la Somme et de la Normandie, de même qu'en Belgique et au Pays-Bas (Antoine et al., 2000). Cette phase d'incision affecte également les vallées de l'Europe du nord-ouest (Haesaerts, 1984 ; Kozarski, 1983 ; Mol et al., 2000 ; Vandenberghe et al., 1987 ; Starkel, 1991). Une évolution comparable est observée dans les fonds de vallée du Bassin parisien, site d'Houdancourt sur l'Oise, de Rochy-Condé sur le Thérain (Pastre et al.. 2003 ) et de la Somme sur le site de Conty (Antoine. 1997a), avec une incision rapide chronologiquement bien établie entre $13 \mathrm{k}$ BP et $12,5 \mathrm{k} \mathrm{BP}$. Cette incision majeure. engendrée par le changement climatique tardiglaciaire, se manifeste sur de nombreux cours d'eau de rang supérieur par une transformation du style fluvial du tressage vers le méandrage (Starkel, 1983). Sur le secteur d'étude, l'incision est associée à un cours d'eau méandriforme dessinant une large boucle relativement symétrique (rayon de courbure entre 550 et $700 \mathrm{~m}$, longueur d'onde de $900 \mathrm{~m}$ ). Néanmoins, cette boucle recoupe en rive gauche un tracé emprunté aujourd'hui par le cours de la Tortue et correspondant au chenal 2 non daté (fig. I et 2). On peut donc envisager un système comportant plusieurs écoulements méandriformes contemporains. Le chenal 2 dont le tracé est effacé de la surface de la plaine a pu être recoupé par le chenal 1 au cours de l'Holocène (fig. 9).

Avec un bassin versant de taille comparable $\left(524 \mathrm{~km}^{2}\right)$ et un débit moyen actuel identique $(5 \mathrm{~m} / \mathrm{s})$, la Selle, affluent de la Somme, constitue un site de comparaison. La rivière s'encaisse au début du Tardiglaciaire de 2 à $3 \mathrm{~m}$ dans les dépôts pléistocènes. Flle développe à la hauteur de Conty plusicurs chenaux totalisant une largeur d'environ $200 \mathrm{~m}$ de large contre environ $70 \mathrm{~m}$ observés dans le chenal 1 à
Houplin-Ancoisne. Le sous-dimensionnement de ce cours par rapport à Conty renforce l'hypothèse de deux chenaux tardiglaciaires pour la Deûle.

\section{Le comblement Bolling initial du chenal}

Le comblement débute avec le dépôt d'une unité grossière sablo-graveleuse (24) qui se distingue des niveaux pléniglaciaires sous jacents par son caractère plus sableux et l'incorporation de passées de limon plastique enrichi parfois de macrorestes végétaux et malacofaunes. Deux interprétations chronologiques sont possibles : (a) le caractère légèrement assymétrique du chenal permet d'assimiler la mise en place de ce niveau à la construction d'un petit banc de convexité inhérent à la dynamique du méandre, dans une section peu sinueuse du chenal. Il s'agirait alors d'un dépôt contemporain de l'incision tardiglaciaire. (b) l'abondance relative des macrorestes végétaux et des malacofaunes pourrait également indiquer un remaniement de la berge ou du fond contemporain du niveau 13. Dans tous les cas, ce dépôt témoigne d'un transit sédimentaire non négligeable durant le début du Bolling. ce que l'on observe également dans la moyenne vallée de l'Oise (Pastre et al., 1997).

Le brusque ralentissement des conditions hydrodynamiques au Bølling se traduit par un colmatage fin sablolimoneux lité (niveau 13, $12560+/-100 \mathrm{BP}$ ) qui tranche avec les sédiments grossiers de l'encaissant pléniglaciaire.

\section{Le développement tourbeux Bølling-Allerod}

Le comblement se poursuit par le dépôt d'un limon organique lité passant latéralement à une tourbe franche (12). Cette évolution latérale de faciès peut être attribuéc aux conditions locales de la chenalisation qui concentre les faibles écoulements (stratifications horizontales du limon organique) tandis que la tourbe se développe en position haute sur les flancs du chenal. En l'absence de donnée chronologique fiable (datation $\langle\mathrm{b}$ » rejetée), cette unité peut être attribuée aussi bien à une seconde partie du Bølling qu'à l'Allerød. Des formations tourbeuses ou de limon tourbeux sont en effet observées dès le Bolling dans de petites vallées du bassin de la Somme, tel le site de Conty dans la vallée de la Selle (Antoine, 1997a), et du bassin de la Seine avec le site de Rochy-Condé dans la vallée du Thérain (Pastre et al., 2003). Daprès 
les auteurs, cette tourbogenèse se limite cependant à certaines sections des petites vallées et aux paléochenaux. Des niveaux tourbeux comparables se développent égálement durant la deuxième partic de l'Allered dans les paléochenaux des rivières du Bassin Parisien. C"est le cas à Béthisy-St-Martin dans la vallée de l'Automne et à Houdancourt dans la vallée de l'Oise (Pastre et al., 2000). De même dans le bassin de la Somme où P. Antoine (1997a) décrit toujours sur le site de Conty un faciès limono-tourbeux à débris végétaux se développant dans plusieurs paléochenaux. Munaut et Paulissen (1973) précisent qu’à l'Allered, une tourbière basse oceupait tout le fond de la vallée de la Petite Nethe en Belgique, tandis que des dépots limono-argileux s'accumulaient dans les zones les plus déprimées. Les mêmes auteurs font état des travaux de Vermeersch (1972) qui a observé dans la vallée de la Winge (Belgique) le développement d'une tourbière durant l'Allerød et le début du Dryas récent. Plus près du site d'étude, dans la vallée de la Scarpe à Douai, Munaut et al. (1995) ont également mis en évidence une séquence Allerød limono-tourbeuse en bordure de chenal tandis qu’a Dourges, dans la partie amont de la vallée de la Deûle, un complexe de tourbe et de limon tuffacé calcaire se développe dans une dépression du fond de vallée au cours de Bolling et de l'Allered (Teheux et Deschodt, 2000).

\section{Première partie du comblement Dryas récent}

Le Dryas récent est identifié à la base par un dépôt limono-sableux lité (9) très riche en malacofaunes brisées. qui constituent de véritables niveaux pluricentimétriques bioclastiques. Au droit de son développement maximum (S11), il intègre de nombreuses lamines organiques avec des macro-restes végétaux. Ce niveau est interprété comme un faciès de remplissage de chenal. II bénéficie de trois dates homogènes (c. d. e. fig. 3) situées entre 10400 et 10600 BP (environ 12900 à 12100 cal. BP. tab. 1). La mise en place du niveau 15 à graviers de craie s"explique par le sapement de la berge concave du chenal. probablement inhérent à l'autoajustement du paléoméandre. Son contact direct sur la tourbe franche (12) et son interstratification avec l'unité 9 (S02) témoignent d'une mise en place rapide contemporaine de la base du dépôt de 9. Le niveau 11 de graviers de craie à matrice limoncuse surmonte directement 15 et est recouvert par 9. Ce faciès résulte probablement de la poursuite d"une érosion plus modérée de la berge, au profil déjà modifice. ou au remaniement des graviers qui en sont issus. Si nous prenons en compte l'oceurrence des dates « e » (10570 BP) dans l'unité 9 et «f » $(10190 \mathrm{BP})$ dans l'unité 11, stratigraphiquement proche. l'intervalle 2 sigma pour cette partie du remplissage se réduit à environ 12350-12150 cal. BP. L'ensemble de ces observations (dépôts plus grossiers à laminations, légère érosion linéaire, sapement de berge), est le signe d'une dynamique fluviale plus active qui reflète la dégradation climatique du Dryas récent. Dans le bassin de la Somme (Antoine. 1997a, 1997b; Antoine et al. 2000) et les vallées du Bassin parisien (Pastre et al., 2000. 2003), cette période est marquée par le colmatage des fonds de vallée sous d’importants volumes de limons calcaires déposés lors des inondations. Le lit mineur contemporain est difficile à repérer. A Conty, son faciès lité à malacofaune et lamines organiques (Antoine, 1997b) présente de fortes similitudes avec l'unité 9 d'Houplin-Ancoine. Toutefois, dans la vallée de la Deûle, les apports sédimentaires semblent beaucoup plus limités et s'arrêtent aux abords immédiats du chenal. Les dépôts sont également beaucoup moins calcaires que ceux rencontrés sur substrat crayeux dans le Bassin parisien et leur volume est insuffisant pour ennoyer une part importante du lit majeur. Ces différences peuvent s'expliquer par l'absence d'affleurement crayeux susceptible de fournir des boues crayeuses : tout le bassin versant est constitué de pentes douces. a l'exception de la Gohelle dans sa partic amont. et est protége par une couverture de loess probablement stabilisée par la pédogenèse Bølling-Allerød et une végétation herbacée (Antoine et al., 2002 ; Pastre et al.. 2002). A Dourges, dans la partie amont de la vallée de la Deûle, le fond de vallée est marqué par un remblaiement modéré sous forme de petites coulées de solifluxion très localisées et par des colluvions limoneuses. Ponctuellement, un démantèlement des formations Bølling-Allerød par des écoulements provenant du versant est associé aux premières colluvions (Teheux et Deschodt, 2000).

A Houplin-Ancoisne, si le bilan sédimentaire de cette partie du Tardiglaciaire tend à un remblaiement du chenal. la faiblesse relative des apports sédimentaires a favorisé une bonne représentation de la part biotique dans la composition des dépôts (débris organiques, coquilles). Plus au nord, en Belgique, des tourbes continuent de se développer au début du Dryas récent dans la vallée de la Wingle (Vermeersch, 1972. in Munaut et Paulissen, 1973) et dans la vallée de la Mark (Vandenberghe et Bohncke. 1985 ; Vandenberghe et al. 1987). Dans ce dernier cas, la recrudescence des inondations participent à l'ensevelissement de la tourbière située sur une basse terrasse pléniglaciaire. A Houplin-Ancoisne, le colmatage du chenal est incomplet, ce qui a permis le développement et la conservation d'un dépôt de la fin du Dryas récent.

\section{Seconde partie du comblement Dryas récent}

La fin du comblement du chenal tardiglaciaire est composé d'une unité sablo-graveleuse (unité 8) et de deux limons gris homogènes (unités 7 et 5) séparés par un niveau discordant à gravicrs de craic anguleux (unité 6). Un petit sol s'est développé au sommet de cette séquence dont l'épaisseur totale est d'environ $85 \mathrm{~cm}$. Aucun élément datable n'a été rencontré et l'occurrence des dates «e » et «f » (10570 et $10190 \mathrm{BP})$ dans les unités 9 et 11 sous-jacentes donnent un terminus post-quem à cette deuxième partie du remplissage de chenal (environ 12350-12150 cal. BP). La stratigraphie est d'une complexité inhabituelle pour le Dryas récent. Toutefois, la géométrie du dépôt détritique, qui l'inclut dans le comblement du chenal tardiglaciaire, l'aspect azoïque de l'ensemble et l'absence de décarbonatation avant le petit sol sommital plaident bien pour une mise en place tardiglaciaire.

L'unité 8 , à graviers de craic roulés, présente une limite inféricure ćrosive qui dessine deux petits chenaux remplis de graviers de craie (sondages 11 et 17, fig. 3). Elle 
tapisse encore le fond d'un chenal évasé et peu profond. La limite représentée par le niveau 6 (déflation ?) présente un léger bombement et marque une modification du profil transversal du chenal. La disparition des structures litées et de la texture sableuse (unités 5 et 7), ainsi que le caractère anguleux des graviers (unité 6), témoignent de la diminution de la dynamique d'écoulement. A ce niveau du colmatage, il est possible que le paléoméandre ne fonctionne plus en tant que tel et soit simplement emprunté par de petits écoulements se concentrant sur les marges du chenal.

La bipartition du Dryas récent observée à HouplinAncoisne est cohérente avec les données paléo-écologiques recueillies dans le bassin de la Somme. Les cortèges de malacofaune y indiquent une première partie froide et humide et une seconde partie moins rigoureuse mais plus sèche (Limondin-Lozouet et Antoine, 2001 ; Limondin-Lozouet et al., 2002). L'aridité de la seconde partie du Dryas récent se traduit plus au nord, en Belgique, aux Pays-Bas et en Allemagne, par la mise en place d'une couverture éolienne envahissant les fonds de vallées (Huisink, 1997, 2000 ; Kasse, 1997 ; Mol, 1997 ; Vandenberghe, 1995 ; Kasse et al., 2003).

\section{4 - FORMATIONS ET ÉVOLUTION HOLOCÈNE}

\section{1 - L'INCISION HOLOCĖNE ET LA FORMATION D'UNE TERRASSE TARDIGLACIAIRE/HOLOCĖNE}

Une deuxième phase d'incision aboutit à la création d'un chenal holocène qui ne se surimpose pas au chenal tardiglaciaire. L'incision est modeste, d'environ $1,5 \mathrm{~m}$ par rapport au sommet des dépôts tardiglaciaires. Dans cette section du cours de la Deûle, elle a probablement suivi le tracé du chenal tardiglaciaire, en étant guidée vers la rive droite par la microtopographie. La berge, qui est composée de sédiment essentiellement sableux et facilement remobilisable (23), a favorisé une érosion latérale expliquant le décalage d'une cinquantaine de mètres en direction de la rive droite (fig. 3). Cette migration latérale a permis la préservation d'une bonne partie des séquences tardiglaciaires. Elle se produit après les dépôts du Dryas récent et avant la datation " $\mathrm{g}$ " $(8380 \pm 50 \mathrm{BP}$, GrA-23190, Boréal). L'encaissement du chenal a donc probablement lieu durant le Préboréal mais sans que l'on puisse l'associer avec certitude au tout début de la période, comme on peut l'observer sur d'autres cours d'eau du nord-ouest de l'Europe (Tebbens et al., 1999 ; Antoine et al., 2000 ; Pastre et al., 2002). La présence d'environ $30 \mathrm{~cm}$ de sable lité gris avec granules de craie à la base du remplissage holocène indique que le chenal est fonctionnel au plus tard jusqu'au Boréal, sauf à considérer que ces dépôts accompagnent une incision tardive.

En rive gauche, l'incision holocène a dégagé une basse terrasse dans les formations tardiglaciaires. En rive droite, le sapement des formations sableuses et loessiques a avivé la terrasse préexistante qui conserve encore aujourd'hui une fraîcheur certaine.

La très basse terrasse tardiglaciaire/holocène de rive gauche a supporté une légère pédogenèse, elle a été ennoyée par des alluvions limoneuses (4) interprétées comme des dépôts d'inondation, puis par des dépôts limono-tourbeux (5). Les deux niveaux (4 et 5) conservent les traces d'une probable intervention anthropique (fossés ?) s'exprimant par des surcreusements localisés (base du niveau 1 entre S16-S22 et sur S24).

Sur le transect (fig. 3), le creusement holocène n'atteint pas le niveau maximal du creusement tardiglaciaire. Il est plus élevé en altitude d'environ $1 \mathrm{~m}$. La section du chenal holocène est de l'ordre de $70 \mathrm{~m}$ de large pour un

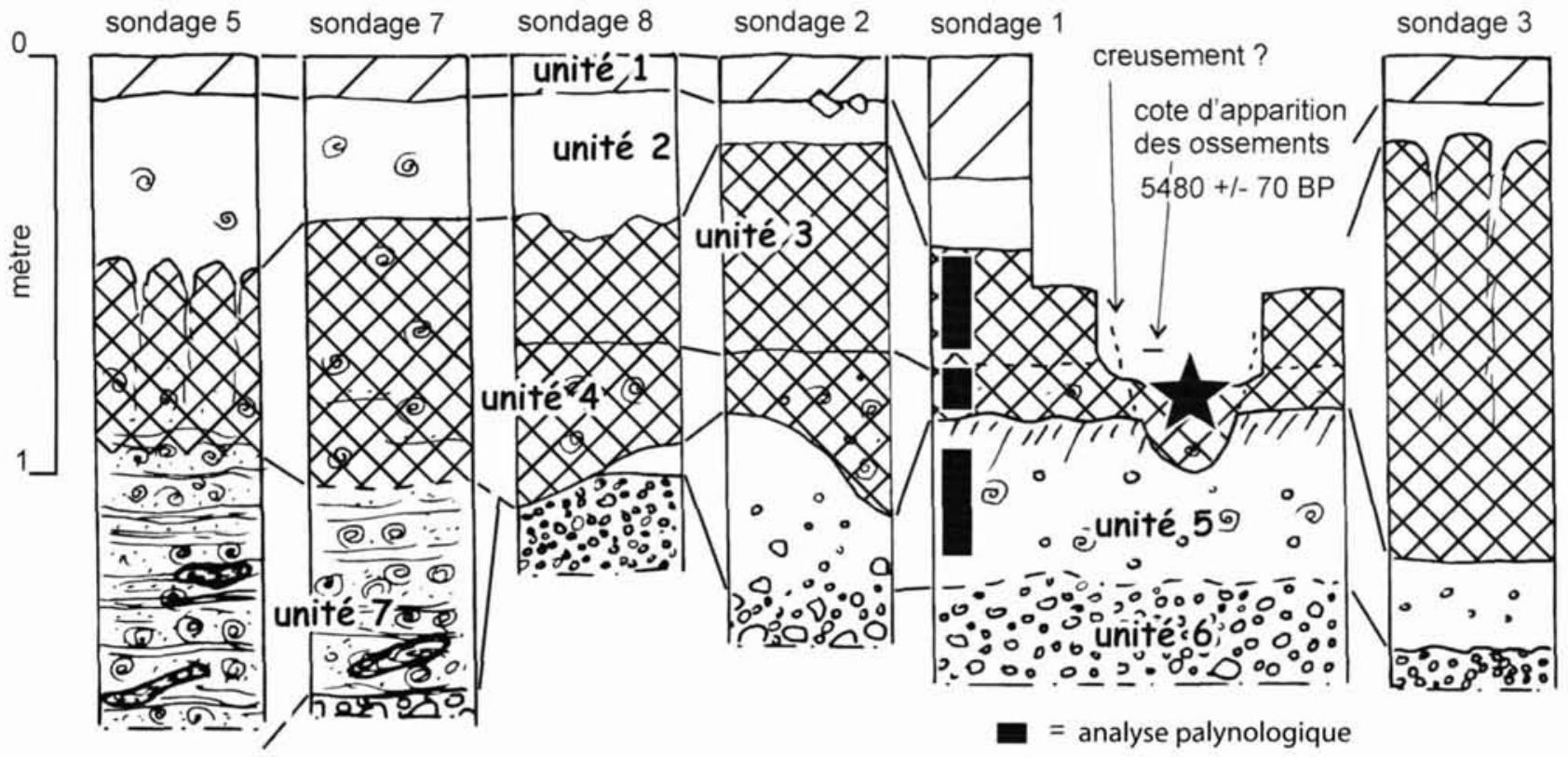

Fig. 6 : Observations en puits au niveau de la station d'épuration. 1 : Labours et remblais ; 2 : limon argileux brun jaune enrichi de grains de craie épars ou de fragments de briques à son sommet (dépôt d'inondations historiques); $3:$ tourbe limoneuse peu épaisse avec fentes de rétraction (sécheresse) ; 4 : limon tourbeux, avec malacofaunes éparses ; 5 : dépôts graveleux bioclastiques riches en bois flotté ; 6 : limon argilo-sableux gris à graviers et malacofaune ; 7 : nappe de graviers de craie.

Fig. 6: Observations at the filtering station, according to a trench. 1: ploughing; 2: brown yellow clayey silt with scattered chalk grains or brick fragments at its top (historical floodplain deposits); 3: silty peat, not very thick, with shrinkage crack (dryness): 4: peaty silt, with scattered malacofaunas; 5: bioclastic gravel deposits with abundant floated wood: 6: gray clayey sandy silt with gravels and malacofaunas: 7 : chalk gravel. 


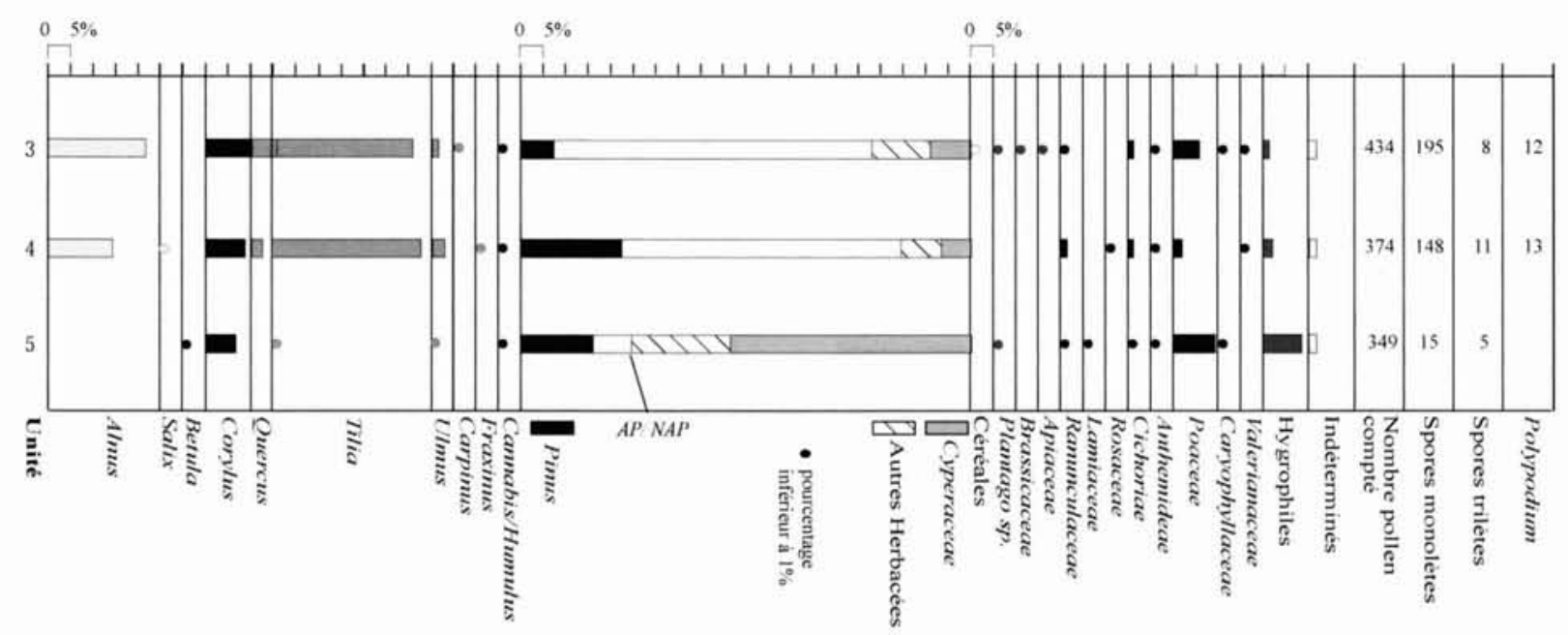

Fig. 7 : Résultats polliniques en histogrammes des niveaux 3, 4 et 5, d'après les observations réalisées en 1999 à la station d'épuration ( $c f$. fig. 6).

Fig. 7: Pollen results in histograms of levels 3.4 and 5, according to the observations carried out in 1999 at the filtering station (cf. fig. 6).

remblaiement total d'environ $2 \mathrm{~m}$ d'épaisseur (débordement du chenal inclus). Avec un bassin versant comparable (cf. supra), la Selle, affluent de la Somme, présente à la hauteur du site de Conty une section plus importante, puisqu'elle atteint un peu plus de $100 \mathrm{~m}$ de large pour environ $3 \mathrm{~m}$ de profondeur (Antoine $e t$ al., 2003). Cette observation renforce la présomption du fonctionnement d'un second chenal plus à l'ouest dans la vallée de la Deûle (fig. 1 et 9).

\section{2 - L'ENVAHISSEMENT TOURBEUX DU LIT MINEUR ET DU FOND DE LA VALLÉE}

Sur le transect, un limon tourbeux lité, à nombreuses malacofaunes remplit la majeure partie du lit mineur (unité 2, fig. 3) jusqu'au moins le Subboréal (datation $« \mathrm{~h} »: 3590 \pm 40 \mathrm{BP}, \mathrm{GrA23286}$ ). Un limon argileux ocre (unité 4 , limon d'inondation), recouvre la rive gauche et scelle la basse terrasse développée dans les formations tardiglaciaires. La fin du comblement se compose d'un limon brun (unité 1) qui envahit tout le profil, au caractère un peu moins tourbeux et avec une malacofaune moins abondante.

En 1999, une série de sondages en puits («SE », fig. 1 et fig. 6) sous la station d'épuration (Deschodt et Créteur, 1999), quelques dizaines de mètres en amont du transect partiel, a permis l'étude des formations holocènes du lit mineur et la réalisation d'analyses palynologiques qui apportent des données complémentaires sur l'Holocène (fig. 7). Sous les formations tourbeuses, l'unité argilosableuse est difficilement attribuable à une chronozone particulière. Peu diversifiée, la végétation majoritaire est avant tout locale et liée aux conditions édaphiques du site. Il s'agit des Cypéracées (53,3\%) et d'un ensemble de plantes aquatiques qui totalisent $8,6 \%$. Cependant, aucune ripisylve n'est mise en évidence. La strate arborée $(\mathrm{AP}=24,4 \%)$ est dominée par le Pin $(16 \%)$, suivi du Noisetier $(6,6 \%)$. On trouve également de façon sporadique quelques pollens de Bouleau, Tilleul et Orme. $\mathrm{La}$ strate herbacée, hormis les hydrophiles et aquatiques, est représentée par les Poacées et la présence de quelques autres taxons, en quantités très faibles et sans signification écologique particulière.

Les unités tourbeuses donnent l'image d'une végétation thermophile où la chênaie mixte est très bien représentée. La très grande extension du Tilleul, corrélée à un enregistrement faible de l'anthropisation, suggère une attribution à la palynozone Atlantique. AP atteint des valeurs autour de $80 \%$. Ce phénomène est imputable à la très grande représentation de la chênaie mixte à Tilleul (plus de $30 \%$ ), Chêne (5,5 et 2,4\%), Orme ( 1,4 et 2,7 \%) et même Charme $(0,7 \%)$. La ripisylve est également bien représentée, essentiellement par l'Aulne (21,7 et 14,2\%). Le Noisetier fait aussi partie des espèces identifiées en nombre avec $9,5 \%$ en moyenne, ainsi que le Pin avec presque $15 \%$ en moyenne. La strate herbacée est relativement peu diversifiée. Elle est composée de Cypéracées, de Poacées, de quelques aquatiques et autres taxons non significatifs. Les indices d'anthropisation y sont quasiment absents si ce n'est deux grains de pollen de céréales dans l'échantillon supérieur.

L'observation de 1999 offre par rapport au transect une unité supplémentaire, non datée, antérieure à la tourbe et située en rive droite (unité 6, fig. 6). Par contre la série tourbeuse avec lits de malacofaune (unité 2, fig. 3) est corrélée sans ambiguïté avec les formations tourbeuses passant à des lits graveleux bioclastiques vers le centre du chenal (unités 3,4 et 7, fig. 6). La partie graveleuse est plus épaisse (plus de $60 \mathrm{~cm}$ ). La palynozone Atlantique proposée pour le développement tourbeux sur le site de la station d'épuration est cohérente avec les datations radiocarbones effectuées sur une structure anthropique reconnue dans les alluvions (fig. 6) ainsi que sur les bois du transect partiel (fig. 3). L'envahissement tourbeux se poursuit sans rupture durant le Subboréal (datation « $\mathrm{h}$ », tab. 1). Il se répand au-delà du lit mineur dans les points bas du fond de vallée, masquant la très basse terrasse tardiglaciaire et participant au développement d'une vaste zone marécageuse d'où émerge des " îlots » loessiques. L'atterrissement de la rive droite était plus avancé en amont, sous la station d'épuration, dans la mesure où une fosse a pu y être creusée durant le Néolithique. 
La stratigraphie holocène observée sur le transect est relativement homogène, avec un remblaiement tourbeux continu. Or, à faible distance, des unités sédimentaires supplémentaires ont été observées (unité 5, fig. 6 ; Deschodt et Créteur, 1999) et des épisodes érosifs d'époque gallo-romaine repérés quelques kilomètres en aval, à Lille, sur des chantiers archéologiques (Deschodt, 1999; Deschodt et Boulen, inédit). Une stratigraphie complexe est également en cours d'exploitation à proximité immédiate du transect (« LMS », fig. 1: Deschodt et Praud, étude en cours). Cette multiplication des observations apportera une meilleure perception de la dynamique holocène, probablement plus complexe et contrastée que celle perçue à travers la seule lecture du transect.

\section{3 - L'ÉVOLUTION HOLOCĖNE DES VERSANTS}

Le transect montre clairement la vigueur de certaines sections de la rive holocène (fig. 3). Cette configuration constitue un facteur attractif pour les occupations humaines : proche de la rivière, l'habitat se réserve un accès facile à l'eau tout en restant à l'abri de la plupart des crues. Les environs de la station d'épuration ont livré plusieurs sites archéologiques, en particulier néolithiques, sur la rive droite du cours de la Deûle. Ils montrent la bonne conservation locale des sols de la première partie de l'Holocène sous des colluvions sableuses (fig. 8). Sur le site de la rue Dormoy (MD fig. 1 et fig. 8), la microtopographie de la basse terrasse loessique a permis la conservation d'un pédon relativement complet dans un léger creux (Deschodt in Fechner et al., 2004). Une pièce isolée du Paléolithique supérieur (détermination E. Martial) a été trouvée au contact de l'horizon humifère et de l'horizon éluvial tandis qu'une pièce du Mésolithique se trouve dans l'horizon humifère. Les structures néolithiques sont comblées par un sédiment sablo-limoneux clair similaire aux colluvions qui recouvrent le sol. Difficile à repérer, leur niveau d'ouverture est au moins pour une partie d'entre elles au sein des colluvions. Ces dernières sont probablement issues de la dénudation de la surface engendrée par l'occupation elle-même (rapide érosion/ accumulation par le ruissellement observé sur le décapage archéologique). Un second horizon humifêre indique une stabilisation du versant. Il a été affecté par le creusement de structures à l'Age du Fer suivi d'une seconde phase de déstabilisation. Chaque épisode d'occupation et d'érosion a remanié une partie des pièces archéologiques plus anciennes. Latéralement et vers le haut du versant, les horizons d'accumulation du sol sont affectés par les labours modernes qui participent, avec les colluvions, au nivellement de la microtopographie de la basse terrasse. Celle-ci présente maintenant une pente régulière vers la Deûle.

\section{5 - CONCLUSION}

Dans un petit bassin versant à fort recouvrement loessique, l'évolution de la Deûle présente des traits originaux et d'autres pouvant être considérés, à l'image

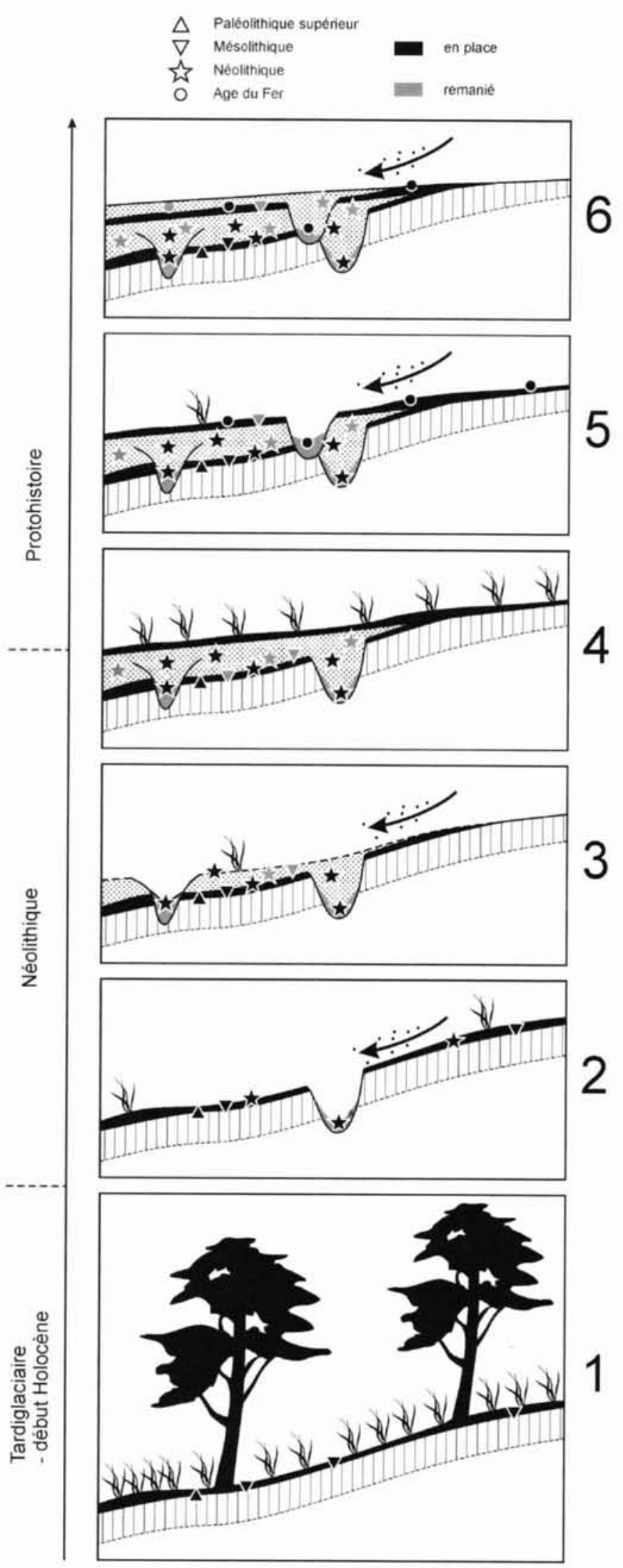

Fig. 8 : Evolution schématique du bas de versant, en rive du lit mineur holocène (fouille archéologique rue Marx Dormoy, en cours d'étude, “ MD » sur fig. 1).

Fig. 8: Sketchy evolution of the slope bottom, on the Holocene river bank (archaeological site under study. Marx Dormoy street, "MD w on fig. (1). 

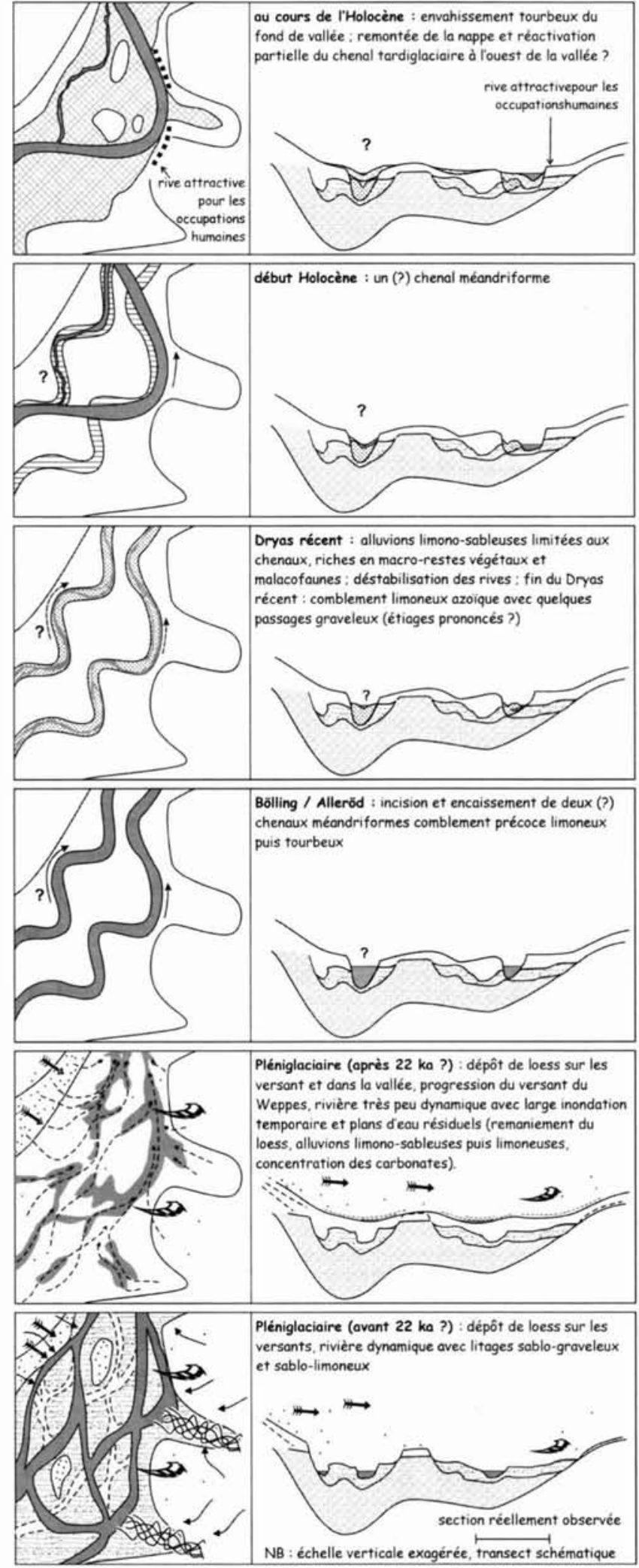

Fig. 9 : Schéma hypothétique de l'évolution paléogéographique de la vallée depuis le dépôt loessique pléniglaciaire.

Fig. 9: Hypothetical diagram of the paleogeographic evolution of the valley since the pleniglacial loess deposit. de sa position géographique, comme intermédiaires entre ceux du Bassin parisien et du Bassin belgo-néerlandais :

1/ A la fin du Pléistocène supérieur, un loess à remaniement fluviatile a envahi le fond très large de la vallée. Il s'agit, en contexte loessique, d'un équivalent des formations limono-sableuses éoliennes et fluvio-éoliennes largement développées en Belgique, aux Pays-Bas et en Allemagne.

2/ L'incision d'un système méandriforme au Bølling dans les formations loessiques et sableuses antérieures crée une forme de terrasse bien marquée similaire à celle des vallées belgo-néerlandaises.

3/ Une sédimentation limoneuse débute au Bølling, suivie d'une tourbe. La majeure partie du comblement est datée du Dryas récent, en deux épisodes distincts. La séquence inférieure est liée au fonctionnement du drain (intercalations organiques et malacofaunes). La séquence supérieure, postérieure à 12350-12150 cal. BP, achève le comblement du chenal dans des conditions plus sèches. Contrairement à la plupart des vallées du nord-ouest européen, les alluvions tardiglaciaires sont confinées au chenal et à ses abords immédiats et ne favorisent pas le développement d'une plaine d'inondation.

4/ L'incision holocène, modeste, se manifeste sous la forme d'un cours d'eau méandriforme jouxtant le chenal tardiglaciaire. Elle développe une très basse terrasse tardiglaciaire/holocène. Contrairement aux vallées belgo-néerlandaises, cet étagement est éphémère et disparaît dans le courant de l'Holocène sous les alluvions tourbeuses. La forme est toutefois distincte et mieux marquée que dans les vallées du Bassin parisien.

5/ L'Holocène se caractérise par un remblaiement tourbeux qui déborde du lit mineur et envahit le fond de vallée. Le paysage actuel est marqué par cette dernière évolution avec une large vallée humide d'où émergent des îlots loessiques. Dans un contexte de relief doux et peu contrasté, la topographie de détail (eau libre, zones légèrement plus élevées) et le développement spatio-temporel des formations tourbeuses sont des clefs nécessaires à la compréhension des sites archéologiques.

Ces résultats découlent de l'observation d'un seul des deux chenaux distingués dans le secteur d'HouplinAncoisne. Il est donc nécessaire d'étendre notre exploration pour comprendre les interrelations entre les deux chenaux. Par ailleurs, des investigations en cours amèneront à compléter une vision encore partielle de l'évolution holocène. Quoi qu'il en soit, ce travail illustre les particularités et le fort potentiel d'enregistrement des vallées du bas-pays du Nord de la France. 


\section{REMERCIEMENTS}

Nous remercions le laboratoire de l'HALMA (Histoire. Archéologic. Littératures des Mondes Anciens). CNRS-UMR 8142, Lniversité de Lille 3, qui a finanes les analyses radiocarbone et le Centrum roor Isotopen Onderzoek de l'Université de Groningen qui les a réalisess. Nous remercions également les membres de l'équipe "Fnvironnements quaternaires continentaux. dynamiques naturelles et anthropisation " de l“UMR 8591-Laboratoire de Géographie Physique de Mcudon pour leurs observations. et plus particulièrement les relecteurs de l'article P. Antoine (UMR 8591) et J.F. Pastre (UMR 8591), pour leurs précicux conseils. Merci a Claire Vanzon pour la relecture de l'abstract.

\section{RÉFÉRENCES}

ANTOINE, P., 1997a - Evolution Tardiglaciaire et début Holocène des vallées de la France septentrionale : nouveaux résultats. Compte Rendu de l'Academie des Sciences de Paris Sciences de la Tente er des planetes, 325, 35-42.

ANTOINE, P., 1997b - Modifications des systèmes fluviatiles a la transition Pléniglaciaire-Tardiglaciaire et à l'Holocène : l'exemple du bassin de la Somme (Nord de la France). Géographie phrsique et Quaternaive, 51. (1).93-106.

ANTOINE, P., 2002 - Les loess en France et dans le Nord-Ouest européen, Revue française de Géotechnique. 99. 3-21.

ANTOINE, P., FAGNART, J.-P., LIMONDIN-LOZOUET, ‥ \& MUNAUT, A.- I, 2000 - Le Tardiglaciaire du bassin de la Somme : éléments de synthèse et nouvelles données. Olaternaire, 11. (2). $85-98$

ANTOINE, P., LALTRIDOU, J.-P., SOMME, J., AUGUSTE, P.. AUFFRET, J.-P., BLAIZE, S., CLET-PELLERIN, M., COLTARD, J.-P., DEW OLF, Y., DUGUÉ, O., JOLY, F., LAIGNEL, B., LAURENT, M., LAVOLLE. M., LEBRET, P., LECOLLE. F. LEFEBVRE, D., LIMONDIN-LOZOUET, N., MUNAUT, A.-V., OZOUF, J.-C., QLESNEL, F. \& ROUSSEAU, D.-D., 1998 - Le. Formations quaternaires de la France du nord-ouest : limites et corrélations, Quaternaire, 9. (3), 227-241.

ANTOINE, P., MUNALT, A.-V., LIMONDIN-LOZOUET, N., PONEL, P. \& FAGNART, J.-P., 2002 - Réponse des milieux de fond de vallée aux variations climatiques (Tardiglaciaire et début Holocène) d'après les données du bassin de la Selle (Nord de la France). Processus et bilans sédimentaires. In J.-P. Bravard et $M$. Magny (dir) "Le's Flentes ont une histoire. Paléoemironnement des rivieres et des lac's firancais depais 15000 ans ". Actes du colloque de Motz, juin 2001. Errance, 15-27.

ANTOINE, P., MUNAUT, A.-V., LIMONDIN-LOZOUET, Y. PONEL, P., DUPERON, J. \& DUPERON, M., 2003 - Response of the Selle River to climatic modifications during the lateglacial and early holocenc (Somme Basin- Nothern France). Quaternam Science Reviews, 22. 2061-2076.

AGENCE DE L'EAL NORD-PICARDIE, 2002 - Données sur l'cau. "Ww: ear-antois-picardic.ti:

BLONDIAU, L., 2002 - Seclin « L'Hôpital », sondages archéologiques, SRA Nord-Pas de Calais, INRAP. rapport non publié.

COLBEAUX, J.-P., LEPLAT, J., PAEPE, R. \& SOMME, J., 1978 Tectonique récente dans le Nord de la France et le Sud de la Belgique : exemple de la Plaine de la Lys (Feuille d'Hazcbrouck à 1/50 000), Annales de la société géologiqué du Nord, tome XV V VIII. séance du 9 mars 1978. 179-188.

DERVILLE, A., 1988 - La maitrise des eaux dans les Pays-Bas français avant Louis XIV. Les Pạs Bas Françass. $13^{\circ}$ Annales, 105-123.

DERVILLE, A., 1990 - Rivières et canaux du Nord/Pas-de-Calais aux époques médiévale et moderne, Revue du Vord. t. LXXII, 284, 5-22.

DESCHODT, L., 1996 - Le Quaternaire de Lambersart « Les Conquérants ", Les Cahiers de la Préhistoire du Vord, 19, $135 \mathrm{p}$.

DESCHODT, L.. 1999 - Ebauche d une cartographic au 1/10 000 des formations superficielles de Lille. Mémoire de DEA sept. 1996. Univ. Lillel, Less Cahiers de Préhistoire du Vord, 21-22, $208 \mathrm{p}$.

DESCHODT, L., 2003 - Houplin-Ancoisne "Le jardin des communautés », rapport intermédiaire : la géologie du site et les premiers résultats de la campagne de sondages à la tarière mécanique. INRAP. rapport non publié. $26 \mathrm{p}$.
DESCHODT, L. \& CRETEUR, Y., 1999 - Houplin-Ancoisne, la station d'épuration. sondages. Document final de synthèse. SRA NordPas de Calais. INRAP, rapport non publié, $17 \mathrm{p}$.

DESSAUX, V., REVILLION, S. \& DELCHAMBRE, S., 1998 Stratégies dexploitation et mutations du paysage dans la valléc de la haute Deulc (Nord) : Iexemple du marais de Seclin du XIlle au XIXe siecle. Revte di Nord, t. LXXX. (326-327). 531-571.

FECHNER, K., BLANCQUAERT, G. \& LANGOHR, R., 2004 L'utilité de l'archéopédologie : l'exemple du site du Marais de Dourges. Archéopages, $\mathrm{n}^{\circ} 12,10-13$.

FRANCHOMME, M., 2003 - Conception ef utilisation d'un $S I G$ pout litude des donnés (paléo-1 emiromementales et archéologiques appligue au basin versant de la haute-Detile. Mémoire de DEA. Univ. Lillel. $118 \mathrm{p}$

HAESAERTS, P., 1984 - Les formations fluviatiles pléistocénes du bassin de la Haine (Belgique). Bulletin de l'Association française pour l'Etude du Quaternaire. 21. 19-26.

HET TE, A., 1886 - Coupe des couches observées dans les travaux de déviation de la Deûle à l'extrémité de l'Esplanade. à Lille, au lieu dit le Petit Paradis (emplacement du siphon), Anmales de la Sociéte Géclogique der Nord, 13,45-48

HUISINK, M., 1997 - Late-glacial sedimentological and morphological changes in a lowland river in response to climatic change: the Maas. southern Netherlands. Journal of Quaternary Science, 12 (3), 209-223

HUISINK, M., 2000 - Changing river styles in response to weichselian climate changes in the Vecht valley, eastern Netherlands. Sedimentar Geolog: 133, 115-134

KASSE, C., 1997 - Cold-climate acolian sand-shect formation in northwestern Furope (c. 14-12.4 ka) : a response to permafrost degradation and increased aridity. Permatiost and Periglacial Process, 8 , 295-311.

KASSE, C., VANDENBERGHE, J., VAN HISSTEDEN, J., BOHNCKE, S.J.P. \& BOS, J.A.A., 2003 - Sensitiv ity of Weichselian fluvial systems to climate change (Nochten mine, eastern Germany). Quaternar. Science Reriens, 22 (2003), 2141-2156.

KOZARSKI, S., 1983 - River channd adjustment to climatic change in vest central Poland, In K. Gregom (ed). Backgrond to Palaeohidrologi: John Wiley, Chichester, 355-374

LADRIĖRE, J., 1885 - Compte-rendu de l'excursion de la Société géologique du Nord aux environs de Lille et considérations sur les terrains quaternaires et récents des vallées de la Lys et de la Deûle, Annales de la Société Géologiane' du Nord, 405-422.

LADRIÈRE, J., 1886 - Le Terrain quaternaire de la vallée de la Deüle, à Lille. comparé à celui du Nord de la France, Annales de la Sociéte Géologique da Nord, 266-286.

LAUTRIDOL, J.-P. \& SOMME, J., 1981 - L'extension des niveauxrepères périglaciaires à grandes fentes de gel de la stratigraphie du Pléistocéne récent dans la France du Nord-Ouest, Bitultyn Pervglacjalm: 28, 179-185

LIMONDIN-I.OZOUET, N., BRIDAULT, A., I.EROYER, C. PONEL, P., ANTOINE, A., CHAUSSÉ, C., MUNAUT, A.-V. \& PASTRE, J.-F., 2002 - Evolution des écosystèmes de fond de valléc en France septentrionale au cours du Tardiglaciaire : l'apport des indicateurs biologiques. In J.-P. Brarard et M. Magny (div) "Les Flewres ont une histoure. Paleoemironnement des rivieres et des lacs fiancais depuis 15000 ans ». Actes du colloque de Motz, juin 2001 Errance, 45-62.

LIMONDIN-LOZOUET, N. \& ANTOINE, P., 2001 - Lateglacial and early holocene palacoenvironmental changes inferred from malacofaunas at Conty (Northern France). Boreas, 30, 148-164

MARTIAL, E., 2003 - Houplin-Ancoisne, rue Gur Mocquet - RD63 nord, rapport de diagnostic archéologique. INRAP, SRA Nord-Pasde-Calais.

MOL, J., 1997 - Fluvial response to weichselian climate changes in the Niederlausitz (Germany). Joumal of Ouatemar. Science. 12 (1) 43-60.

MOL, J., VANDENBERGHE, J. \& KASSE, C., 2000 - River responso to variations of periglacial climate in mid-latitude Europe. Geomorphologt: 33, 131-148.

MUNAUT, A.-V. \& PAULISSEN, E., 1973 - Evolution et paléo-écologie de la vallée de la Petite Nèthe au cours du post-Würm (Belgique) Annales de la Société Géologiane' de Be/gique. t. 96, 301-348 
MUNAUT, A.-V., SOMMÉ, J. \& DEMOLON, P., 1995 - Tardiglaciaire (Alleröd) et Holocène ancien dans la vallée de la Scarpe à Douai (Nord de la France), Quaternaire, 6, (3-4), 167-172.

PAEPE, R., 1965 - Evolution morphologique de la Plaine de la Lys et des Monts des Flandres. Bulletin de la Société belge de Géologie, de Palcontologie et d\% Hrologie. 74, $121-1,38$.

PAEPE, R. \& SOMMÉ, J., 1970 - Les loess et la stratigraphie du Pléistocéne récent dans le Nord de la France et en Belgique. Annales de la Société Géologique du Nord, XC, 4, 191-201.

PAEPE, R. \& VANHOORNE, R., 1967 - The Stratigraphy and Palaeobotany of the Late Pleisfocene in Belgium. Memoire pour servir à l'explication des Cartes géologiques et minières de la Belgique, $n^{\circ} 8$. Ministère des affaires économiques, administration des mines, Service Géologique de Belgique 1967, 96 p., 14 pl.

PASTRE, J.-F., FONTUGNE, M., KLZUCUOGLU, C., LEROYER, C., LIMONDIN-LOZOUET, N., TALON, M. \& TISNERAT, N. 1997 - L'évolution tardi- et postglaciaire des lits fluviaux au nord-est du bassin de Paris (France). Relations avec les données paléoenvironnementales et l'impact anthropique sur les versants. Géomorphologie : relief. processus, emironnement, 4, 291-312.

PASTRE, J.-F., LEROYER, C., LIMONDIN-LOZOUET, N., CHAUSSE, C., FONTUGNE, M., GEBHARD'T, A., HATTE, C. \& KRIER, V., 2000 - Le Tardiglaciaire des fonds de vallée du Bassin Parisien, Quatemaire, 11,(2), 107-122.

PASTRE, J.-F, LEROYER, C., LIMONDIN-LOZOUET, N., ORTH, P., CHAUSSÉ, C., FONTLGNE, M., GALTHIER, A., KUNESCH, S., LE JEUNE, Y. \& SAAD, M.-C.. 2002 - Variations paléoenvironnementales et paléohydrologiques durant les 15 derniers millénaires : les réponses morphosédimentaires des vallées du Bassin Parisien (France). In J.-P. Bravard et M. Masny (dir:) "Les Fleaves ont ane histoire. Paleonniromement des rivienes at des lac français depuis 15000 ans ". Actes du colloque de Motz. juin 2001, Errance. 29-44.

PASTRE, J.-F., LIMONDIN-LOZOUET, N., LEROYER, C. PONEL, P. \& FONTUGNE, M., 2003 - River system evolution and environmental changes during the Lateglacial in the Paris Basin (France). Quaternary Science Reviens, 22, 2177-2188.

RÉVILLION, S., 1988 - La collection Louis Théry à Lille (deuxièmo partic), Revue du Nord, 70, 123-173.

SCHWAN, J., 1986 - The origin of horizontal alternating bedding in weichselian aeolian sands in Northwestern Europe. Sedimentary Geology, 49, 73-108

SOMMÉ, J., 1967 - Tectonique récente dans la région de Lille : pays de Weppes et Mćlantois occidental, Revue de Gémo'phologit' Dynamique, $\mathrm{n}^{\circ} 2,1967,11 \mathrm{p}$.

SOMME, J., 1968 - Coupe dans le Quaternaire récent de la vallée de la Deûle, près de Lille. Bulletin de l'Association française pour l'étude du Quaternaire, 2, 89-99.

SOMMÉ, J., 1969 - Stratigraphie des limons de la région du Nord de la France (Flandre-Artois), Bulletin de l'Association francaise pour l'étude dur Quatemaire, 71-78.
SOMMÉ, J., 1977 - Les plaines du Nord de la France et leur bordure. Etude géomorphologique. Thèse d'Etat, Univ. Paris I, 1975, Paris Champion, 2 vol. : 1-810, 185 fig.

SOMMÉ, J., MUNAUT, A.-V., PUISSÉGUR, J.-J.. CUNAT-BOGÉ, i. HEYVAERT, F \& LEPI.AT, J., 1996 - L'Eémien sous les formations fluviatiles weichsélicnnes et holocènes du sondage d'Erquinghem (Nord de la France) dans la Plaine de la Lys. Quaternaire, 7. (1). 1996, 15-28.

STARKEL, L., 1983 - The reflection of hydrologic changes in the fluvial environment of the temperate zone during the last 15000 years. In Background to Palaeohydrology, John Wiley ed., Chichester, 213-235

STARKEL, L., 1991 - Long-Distance Correlation of Fluvial Events in the Temperate Zone. In L. Starkel, K.J. Gregoml. J.B. Thornes eds. Temperate Palacohydrology, Wiley, 473-491.

TEBBENS, L., VELDKAMP, A., WESTERHOFF, W. \& KROONENBERG, S.-B., 1999 - Fluvial incision and channel downcutting as a response to Lateglacial and Early Holocene climate change: the lower reach of the River Meuse (Maas), The Netherlands, Journal of Quaternary Science, 14.59-75

TEHEUX, E. et DESCHODT, L., 2000 - Dourges, plate-forme multimodale. Rapport des éraluations paléolithiques à Hénim-Bealtmomt. Sites n 62427025 et 62427027AH. Rapport non publić, SRA. AFAN, 13 p.

VALLIN, L., 1991 - La Flandre intérieure et la métropole Lilloise, Cahiers de la Préhistoire du Nord, 10, 26-32.

VANDENBERGHE, J., 1985 - Paleocnvironment and stratigraphy during the Last Glacial in the belgian-dutch border region, Quaternary Research, 24, 23-38.

VANDENBERGHE, J., 1995 - Timescales, climate and river development, Quaternary Science Reviews, 14, 631-638.

VANDENBERGHE, J., 2001 - A typology of Pleistocene cold-based rivers, Quaternary International, 79 (2001), 111-121.

VANDENBERGHE, J. \& BOHNCKE, S., 1985 - The weichselian late glacial in a small lowland valley (Mark river, Belgium and The Netherlands), Bulletin de I'Association Francaise pour l'Etude du Quaternaire, 2-3, 167-175

VANDENBERGHE, J., BOHNCKE, S., LAMMERS, W. \& ZILVERBERG, L., 1987 - Geomorphology and palaeoecology of the Mark valley (southern Netherlands): Geomorphological valley development during the Weichselian and Holocene. Boreas, 16, 55-67.

VANDENBERGHE, J. \& WOO, M.-K., 2002 - Modern and ancient periglacial river types. Progress in Phwsical Geography, 26 (4), $479-506$.

VALLIN, L., 1990 - La Flandre intérieure et la métropole Lilloise, Cahiers de la Préhistoire du Nord, n 10, 1991, 26-32.

VAN HUISSTEDEN, J. \& VANDENBERGHE, J., 1988 - Changing fluvial style of periglacial lowland rivers during the Weichselian Pleniglacial in the eastern Netherlands, Z. Geomorph. N.F., 131-146.

VERMEERSCH, P., 1972 - Twee Mesolitische sites te Holsbeek, Awhaeologia Belgica. 138. $141 \mathrm{p}$. 